\title{
Molecular Simulations of Adsorption Isotherms for Linear and Branched Alkanes and Their Mixtures in Silicalite
}

\author{
T. J. H. Vlugt, R. Krishna, and B. Smit* \\ Department of Chemical Engineering, Universiteit van Amsterdam, Nieuwe Achtergracht 166, \\ 1018 WV Amsterdam, The Netherlands
}

Received: June 23, 1998; In Final Form: October 21, 1998

The configurational-bias Monte Carlo (CBMC) technique has been used for computing the adsorption isotherms for linear and branched 2-methylalkanes on silicalite. The carbon numbers of the alkanes ranged from four to nine. For branched alkanes inflection behavior was observed for all carbon numbers studied. The inflection was found to occur at a loading of four molecules per unit cell. Below this loading the branched alkanes are seen to be located predominantly at the intersections of the straight and zigzag channels. To obtain loadings higher than four, the branched alkane must seek residence in the channel interiors which is energetically more demanding and therefore requires disproportionately higher pressures; this leads to the inflection behavior. Linear alkanes with six and more carbon atoms also were found to exhibit inflection behavior. Hexane and heptane show inflection due to commensurate "freezing"; the length of these molecules is commensurate with the length of the zigzag channels. This leads to a higher packing efficiency than for other linear alkanes. Available experimental data from the literature are used to confirm the accuracy of the predictions of the CBMC simulations. Furthermore, the temperature dependency of the isotherms are also properly modeled. For purposes of fitting the isotherms we found that the dual-site Langmuir model provides an excellent description of the simulated isotherms for linear and branched alkanes. In this model we distinguish between two sites with differing ease of adsorption: site A, representing the intersections between the straight and zigzag channels, and site $\mathrm{B}$, representing the channel interiors. CBMC simulations of isotherms of 50-50 binary mixtures of $\mathrm{C}_{5}, \mathrm{C}_{6}$, and $\mathrm{C}_{7}$ hydrocarbon isomers show some remarkable and hitherto unreported features. The loading of the branched isomer in all three binary mixtures reaches a maximum when the total mixture loading corresponds to four molecules per unit cell. Higher loadings are obtained by "squeezing out" of the branched alkane from the silicalite and replacing these with the linear alkane. This "squeezing out" effect is found to be entropic in nature; the linear alkanes have a higher packing efficiency and higher loadings are more easily achieved by replacing the branched alkanes with the linear alkanes. The mixture isotherms can be predicted quite accurately by applying the appropriate mixture rules to the dual-site Langmuir model. This model allows the mixture isotherm to be predicted purely on the basis of the parameters describing the isotherms of the pure components. The sorption selectivity exhibited by silicalite for the linear alkane in preference to the branched alkane in mixtures of $\mathrm{C}_{5}, \mathrm{C}_{6}$, and $\mathrm{C}_{7}$ hydrocarbon isomers, provides a potential for the development of a novel separation technique based on entropy-driven sorption selectivity.

\section{Introduction}

Detailed knowledge of the adsorption of hydrocarbons in zeolites is of considerable practical interest in petrochemical applications. ${ }^{1}$ Adsorption isotherms provide information on the amount of hydrocarbons adsorbed in these porous materials at a given pressure and temperature. Recent studies have revealed some interesting characteristics of the adsorption isotherms of hydrocarbons. For example, the isotherms of most linear hydrocarbons in the zeolite silicalite show simple Langmuir behavior. ${ }^{2}$ The isotherms of heptane and hexane, however, show an inflection point. Evidence of this surprising inflection behavior can be gleaned by careful analysis of experimental data from various sources. ${ }^{3-6}$ More recent and systematic studies have confirmed this peculiar behavior of hexane and heptane. ${ }^{7-12}$ It is interesting to note that computer simulation studies had preceded these experimental works with a possible explanation

* Author to whom correspondence should be addressed. of this behavior in terms of a commensurate freezing of hexane and heptane in the zigzag channels of silicalite. ${ }^{13}$

Fewer experimental data are available for adsorption of branched hydrocarbons adsorbed in silicalite. The adsorption isotherms of isobutane also showed an inflection, ${ }^{14-16}$ but for 2-methylpentane a simple Langmuir isotherm was found. ${ }^{17}$ Molecular simulations have shown that the inflection of isobutane is related to the preferential adsorption of the branched alkanes at the intersections of the zigzag and straight channel of silicalite. ${ }^{15}$

Experimentally, the determination of adsorption isotherms of long-chain alkanes can be time-consuming. For example, Stach et al. ${ }^{18}$ report that measurement of each isotherm for decane in silicalite requires at least two weeks of equilibration. It is therefore an important question whether molecular simulations provide an attractive alternative for estimating the adsorption of long-chain hydrocarbons in the pores of a zeolite. The main reason experimentally it takes two weeks to achieve equilibration is that the diffusion of long-chain alkanes is very slow. Such 
slow diffusion would lead to extremely long simulation times if the conventional molecular dynamics or Monte Carlo simulation techniques were to be used. ${ }^{19}$ The configurational-bias Monte Carlo technique has been developed to reduce these simulation times many orders of magnitude. ${ }^{13,20,21}$

For mixtures of hydrocarbons adsorption isotherms for the short-chain alkanes have been presented, ${ }^{22,23}$ but to the best of our knowledge data on mixtures of long-chain alkanes and branched alkanes are lacking. The experimental determination of a mixture isotherm involves not only measuring the weight increase of the zeolite as a function of pressure but also the change in composition of the gas mixture. Therefore mixture isotherms are significantly more complicated to measure compared to a pure component isotherm. If computer simulations could be used to obtain good estimates of such isotherms, this would be of considerable importance since most practical applications involve mixtures of hydrocarbons.

In this work we present the results of computer simulation of linear and branched alkanes and their mixtures in the zeolite silicalite. We focus on the development of the model and a detailed comparison with experimental data for the linear and branched alkanes. In addition we demonstrate that these isotherms can be described quantitatively with a dual-site Langmuir isotherm. We show that this dual-site model, with appropriate mixing rules, can also be used to make a reasonable prediction of the mixture isotherms.

We continue this article with a section describing the details of the model and how the parameters of the potential have been optimized. In Section 3 some details on the simulations are given, and in Sections 4, 5, and 7 the results of the simulations of the linear, branched, and mixtures are presented, respectively.

\section{Model}

In practical applications of the adsorption of hydrocarbons in zeolites, the temperatures and pressures of interest can vary significantly. It is therefore important that the models for the hydrocarbon and zeolites give reasonable results for the thermodynamics over a wide range of temperatures and pressures.

The linear and branched alkanes are described with a unitedatom model, i.e., $\mathrm{CH}_{3}, \mathrm{CH}_{2}$, and $\mathrm{CH}$ groups are considered as single interaction centers. ${ }^{24}$ The bonded interactions include bond-bending and torsion potentials, the nonbonded interactions are described with a Lennard-Jones potential. A way to obtain reasonable Lennard-Jones parameters is to fit the LennardJones parameters to reproduce the vapor-liquid curve of the phase diagram. In ref 25 it is shown that the prediction of the vapor-liquid curve is very sensitive to the choice of the nonbonded Lennard-Jones potential. The model of Siepmann et al. ${ }^{26}$ can describe the vapor-liquid curves of a large number of alkanes over a large temperature range. This model has been further refined and extended to branched alkanes in refs 27 , 28. We have compared the different sets of parameters to investigate how sensitive the adsorption of hydrocarbons in zeolite is for these parameters. This comparison indicates that the results do not differ significantly and for all tested sets gave a very good prediction of the vapor-liquid curves. The details of the alkane model we have used in this work are given in Appendix A.

Following Kiselev and co-workers, ${ }^{29}$ the zeolite is modeled as a rigid crystal. This allows the use of interpolation techniques to determine the interaction of an alkane atom with the zeolite and avoids having to consider all zeolite atoms. ${ }^{21,30}$ The interactions of the alkane atoms with the zeolite atoms are dominated by the dispersive interactions with the oxygen

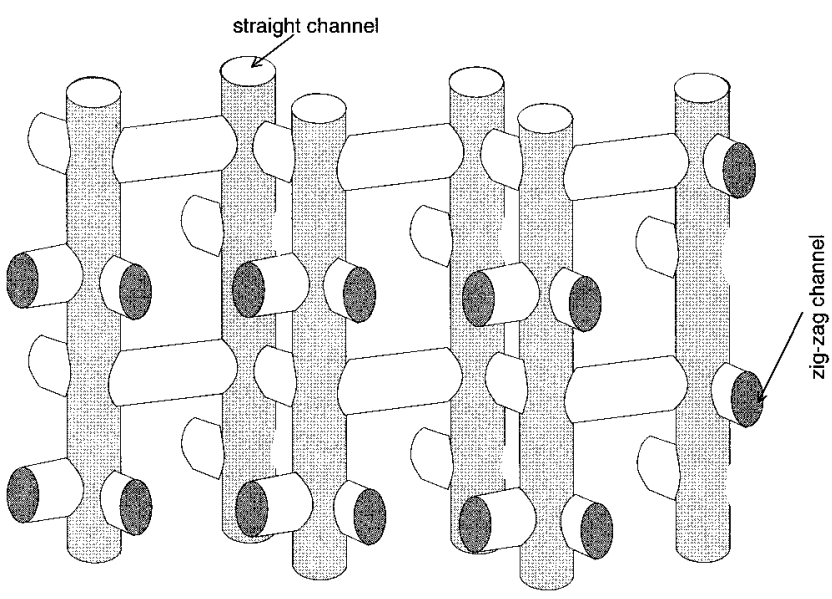

Figure 1. Schematic drawing of the pore structure of silicalite.

TABLE 1: Lennard-Jones Parameters for the Zeolite-Alkane Interactions of the Model Proposed by June et al. $^{30}$ and the Model Developed in This Work

\begin{tabular}{lccccc}
\hline & $\sigma_{\mathrm{CH}_{\mathrm{i}} \mathrm{O}} /$ & $\epsilon_{\mathrm{CH}_{\mathrm{O}} \mathrm{O}} /$ & $\epsilon_{\mathrm{CH}_{2} \mathrm{O}} /$ & $\epsilon_{\mathrm{CH}_{3} \mathrm{O}} /$ & $\epsilon_{\mathrm{CH}_{4} \mathrm{O}} /$ \\
& $\AA$ & $k_{\mathrm{B}} / K$ & $k_{\mathrm{B}} / K$ & $k_{\mathrm{B}} / K$ & $k_{\mathrm{B}} / K$ \\
\hline June et al. & 3.364 & & 83.8 & 83.8 & \\
this work & 3.60 & 58.0 & 58.0 & 80.0 & 96.5
\end{tabular}

TABLE 2: Parameters for the torsion potential of the branched alkanes, ${ }^{70}$ a $\mathrm{CH}_{3}$ group connected to a $\mathrm{CH}$ group is denoted by $\mathrm{CHb}_{3}$, the letter $i$ is used to indicate either a $\mathrm{CH}_{3}$ or $\mathrm{CH}_{2}$ group, i.e., $i=2,3^{a}$

\begin{tabular}{lcccc}
\hline & $C_{0} / k_{\mathrm{B}} / K$ & $C_{1} / k_{\mathrm{B}} / K$ & $C_{2} / k_{\mathrm{B}} / K$ & \multicolumn{1}{c}{$C_{3} / k_{\mathrm{B}} / K$} \\
\hline $\mathrm{CH}_{i}-\mathrm{CH}_{2}-\mathrm{CH}-\mathrm{CHb}_{3}$ & 373.0512 & 919.0441 & 268.1541 & -1737.2160 \\
$\mathrm{CH}_{i}-\mathrm{CH}_{2}-\mathrm{CH}_{2}-\mathrm{CH}$ & 1009.728 & 2018.446 & 136.341 & -3164.520 \\
$\mathrm{CH}_{i}-\mathrm{CH}_{2}-\mathrm{CH}_{2}-\mathrm{CH}_{i}$ & 1009.728 & 2018.446 & 136.341 & -3164.520
\end{tabular}

${ }^{a}$ In case of a $\mathrm{CH}$ group the total torsion potential is the sum of two contributions.

TABLE 3: Parameters for the Lennard-Jones Potential Describing the Interactions between Pseudo atoms of a branched alkane ${ }^{a, 27}$

\begin{tabular}{lcc}
\hline & $\epsilon / k_{\mathrm{B}}(\mathrm{K})$ & $\sigma(\AA)$ \\
\hline $\mathrm{CH}_{4}-\mathrm{CH}_{4}$ & 148 & 3.73 \\
$\mathrm{CH}_{3}-\mathrm{CH}_{3}$ & 98.1 & 3.77 \\
$\mathrm{CH}_{2}-\mathrm{CH}_{2}$ & 47.0 & 3.93 \\
$\mathrm{CH}-\mathrm{CH}$ & 12.0 & 4.1
\end{tabular}

${ }^{a}$ We have also given the parameter for the methane-methane interactions. ${ }^{86}$

atoms, ${ }^{29}$ these interactions are described with a Lennard-Jones potential. In ref 31 it is shown that to describe an adsorption isotherm sufficiently accurately, it is important to have models that yield an accurate prediction of both the Henry coefficient and the heat of adsorption. For the short-chain alkanes there is sufficient experimental data to arrive at a reasonably reliable model, for the long-chain alkanes, however, there is far less experimental data, which makes it difficult to perform a careful test of the model.

To reduce the set of interaction parameters, we have assumed that the size parameter of the Lennard-Jones potential $(\sigma)$ is equal for all pseudo atoms including methane, ethane, and propane. However, one would expect that all size parameters are different. Because a united-atom force field implies lumping of parameters it is very difficult to justify values of parameters based on reasons other than a good reproduction of experimental data, so the choice of equal $\sigma$ is justified. This has as additional advantage that the same interpolation table can be used for all interactions. In Table 1 the parameters of the Lennard-Jones 
potential are given of two models that we have used in this study. These parameters have been chosen such that a reasonable prediction of the Henry coefficient and heats of adsorption is presented. In Section 4A the details on these calculations are given.

A schematic drawing of the silicalite structure is shown in Figure 1. Silicalite has two types of channels, straight and zigzag channels which are connected via intersections.

\section{Simulation Technique}

In this work we have used NVT Monte Carlo simulations in combination with the configurational-bias Monte Carlo technique ${ }^{19,32-35}$ to determine the heat of adsorption and the Henry coefficient. ${ }^{20,21}$ The adsorption isotherms have been determined using grand-canonical Monte Carlo simulations, also in combination with the configurational-bias Monte Carlo technique. The configurational-bias Monte Carlo technique is essential for simulating long-chain alkanes. ${ }^{19}$ The technical details of these methods are described in refs 19, 21, 36, below a short description is given. Further applications of this simulation technique can be found in refs $37-41$.

The simulations are performed in cycles, in each cycle an attempt is made to perform one of the following moves:

(1) displacement of a chain; a chain is selected at random and given a random displacement. The maximum displacement was taken such that $50 \%$ of the moves were accepted.

(2) rotation of a chain; a chain is selected at random and given a random rotation around the center of mass. The maximum rotation was selected such that $50 \%$ of the moves were accepted.

(3) partial regrowing of a chain; a chain is selected at random and part of the molecule is regrown using the configurationalbias Monte Carlo scheme. It is decided at random which part of the chain is regrown and with which segment the regrowing is started. For branched alkanes there is some confusion in the literature how to grow these molecules. In Appendix B2 we discuss the various approaches.

(4a) regrowing of the chain (only for the case of NVTsimulations); a chain is selected at random and is completely regrown at a randomly selected position. During this step, data is collected from which the Henry coefficient is determined.

(4b) exchange with reservoir (only in the case of grandcanonical simulations); it is decided at random whether to add or to remove a molecule from the zeolite. This exchange with the reservoir is done using the configurational-bias Monte Carlo scheme. In ref 36, a detailed derivation of the acceptance rules is given. In this derivation the reference state has been introduced incorrectly. In Appendix B1 the correct reference state is derived.

(4c) change of identity (only in the case of mixtures); one of the components is selected at random and an attempt is made to change its identity. ${ }^{42}$ The acceptance rules for this type of move are given in ref 43 .

The relative probabilities for attempting these moves were such that in the NVT-simulations $10 \%$ of the total number of moves were displacements, $10 \%$ rotations, $10 \%$ partial regrowths, and $70 \%$ regrowths of the entire molecule. For the case of grand-canonical simulations of the pure components, the distribution of moves was $15 \%$ displacements, $15 \%$ rotations, $15 \%$ partial regrowths, and 55\% exchanges with the reservoir. For the mixture, the number of exchanges was reduced to $50 \%$ and the remaining $5 \%$ of the moves were attempts to change the identity of a molecule. The number of trial orientations in the configurational-bias Monte Carlo scheme was six for all

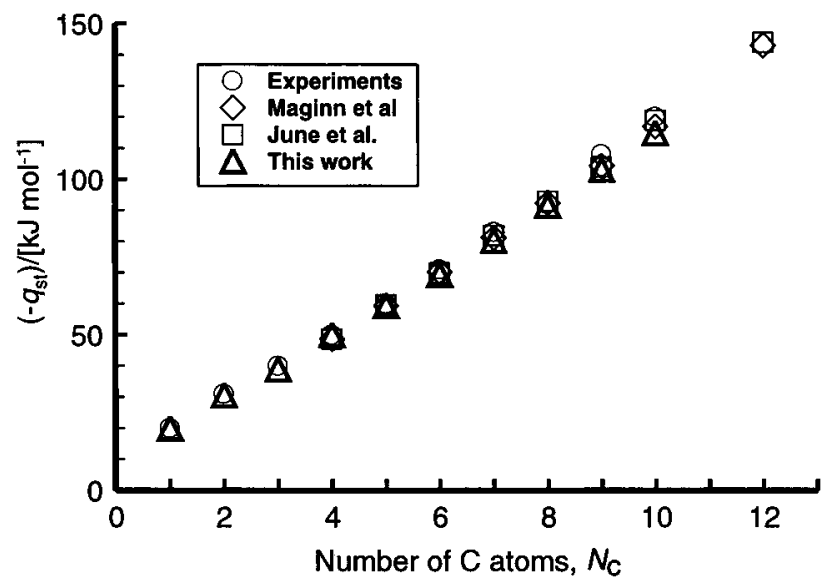

Figure 2. Heats of adsorption $\left(-q_{\mathrm{st}}\right)$ as a function of the number of carbon atoms $N_{\mathrm{c}}$ of the alkanes adsorbed in silicalite.

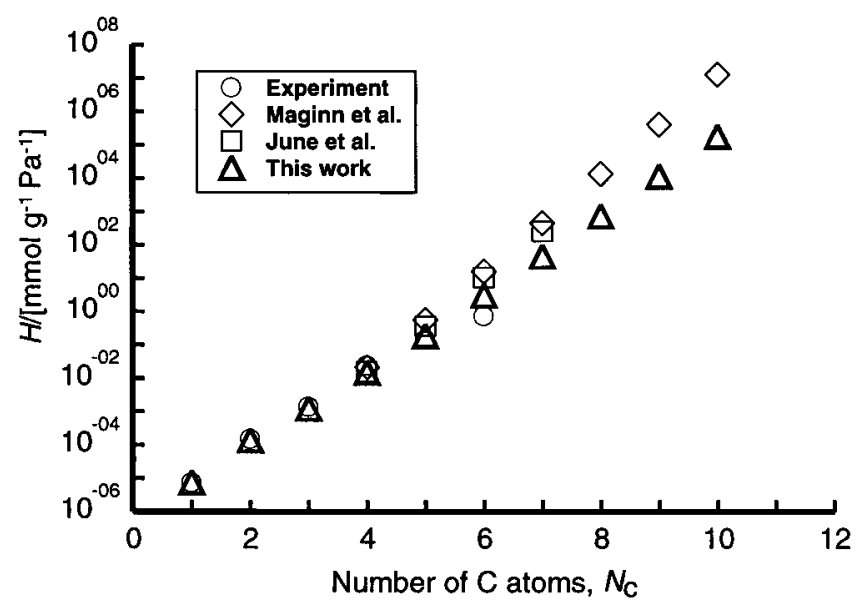

Figure 3. Henry coefficients, $H$, (in mmol g $\mathrm{g}^{-1} \mathrm{~Pa}^{-1}$ ) of linear alkanes as a function of the number of carbon atoms $N_{\mathrm{c}}$ in silicalite.

molecules. In addition, we used the multiple first-bead scheme ${ }^{44}$ with 15 trail positions for the first bead. For the NVTsimulations the total number of cycles was at least $10^{6}$. In a cycle, the number of trial moves is equal to the number of particles with a minimum of 20 trial moves per cycle. The grandcanonical simulations were started from the end configuration of a simulation at a lower chemical potential. We have allowed at least $10^{5}$ cycles for equilibration, and subsequent production runs were at least $10^{5}$ cycles. For the longest chains and at high loading a larger number of cycles were performed. A more detailed description of the program including various tricks to increase the speed of the calculation is given in ref 45 and can be found on the Web. ${ }^{46}$

\section{Linear Alkanes}

A. Heats of Adsorption and Henry Coefficients. To test our model we use the experimental heats of adsorption and Henry coefficients of the linear and branched alkanes.

In Appendix $\mathrm{C}$ a compilation of the experimental data is given. In Figure 2 the experimental heats of adsorption are compared with the results from simulations using the models given in Table 1. Both the model of June et al. ${ }^{30}$ and the model introduced in this work reproduce the experimental data. In addition, this figure also shows that our simulation results are in excellent agreement with the configurational-bias Monte Carlo integration calculation of Maginn et al. ${ }^{47}$

Figure 3 compares the experimental Henry coefficients with the predictions of the various models. For the Henry coefficient 


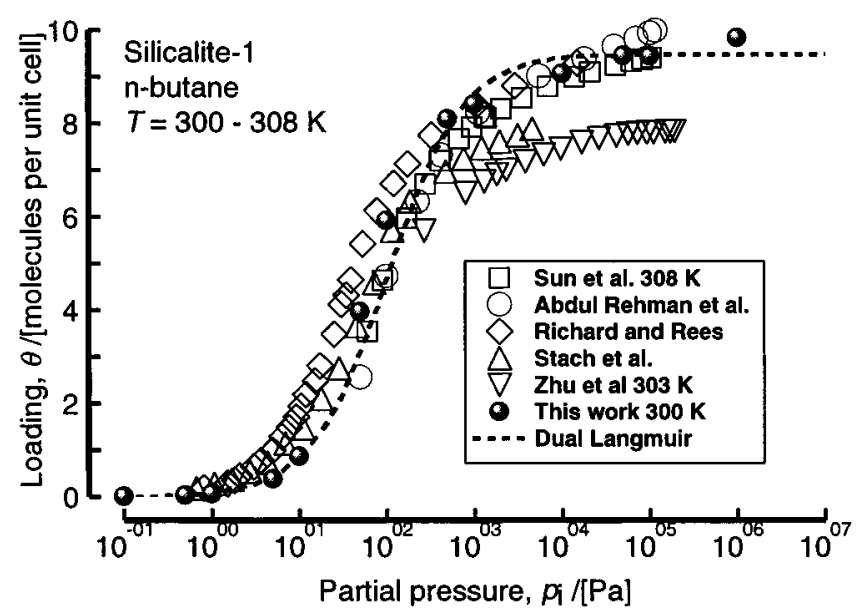

Figure 4. Comparison of adsorption isotherms of butane in silicalite.

there is a significant difference between the various models. Note that the results are plotted on a logarithmic scale, a small deviation from the experimental value gives already a significant deviation for the adsorption isotherms. The results indicate that the model of June et al. ${ }^{30}$ gives a good description for butane, but deviates significantly for the higher alkanes. Our model describes the short-chain alkanes very well but deviates, although less than the model of June et al., for hexane and the longer alkanes. For both models, the simulation data for the Henry coefficients fall on a straight line. The experimental data, however, suggest that the Henry coefficients deviate from a straight line for the longer alkanes. We have also calculated the Henry coefficients for various other sets of parameters but always obtained a straight line. Although we did not test all combinations of parameters, these results indicate that with the current set of models one cannot describe this deviation from a straight line. It would be interesting to investigate whether a straight line is also observed in a simulation with a flexible zeolite lattice.

B. Adsorption Isotherms. The adsorption isotherms of methane, ethane, and propane as predicted by the model developed in this work are reported in ref 48. In the tested temperature range $T=275-350 \mathrm{~K}$ the model reproduces the experimental isotherms very well. For butane the simulation results are compared in Figure 4 with experimental data of Abdul-Rehman et al., ${ }^{22,49}$ Richard et al., ${ }^{50}$ Stach et al., ${ }^{2}$ Sun et al., ${ }^{9}$ and Zhu et al. ${ }^{14}$ The simulation results are in good agreement with the experimental data. The maximum loading of Zhu et al. is considerably lower than the maximum loading of the other isotherms.

The simulated adsorption isotherm of pentane is compared in Figure 5 with the experimental isotherms of Rakhmatkariev et al., ${ }^{4}$ Dubinin et al., ${ }^{5}$ and Sun et al. ${ }^{9}$ The experimental data differ significantly. The maximum loading obtained by Sun et al. is significantly higher than the maximum loading obtained by Rakhmatkariev et al. and Dubinin et al. The maximum loading of Sun et al. agrees very well with the maximum loading obtained from the simulations. A similar agreement with the data of Sun et al. and our simulation results for the maximum loading is obtained for butane (see Figure 4) and hexane (see Figure 6). For these systems more experimental data is available which is consistent with the data of Sun et al. This suggests that the silicalite used by Rakhmatkariev et al. and Dubinin et al. may suffer from pore blocking.

In Figure 6 the experimental isotherms for hexane of Stach et al., ${ }^{2}$ Richard and Rees, ${ }^{6}$ and Sun et al. ${ }^{9}$ are compared with the simulation results using the model of June et al. and the

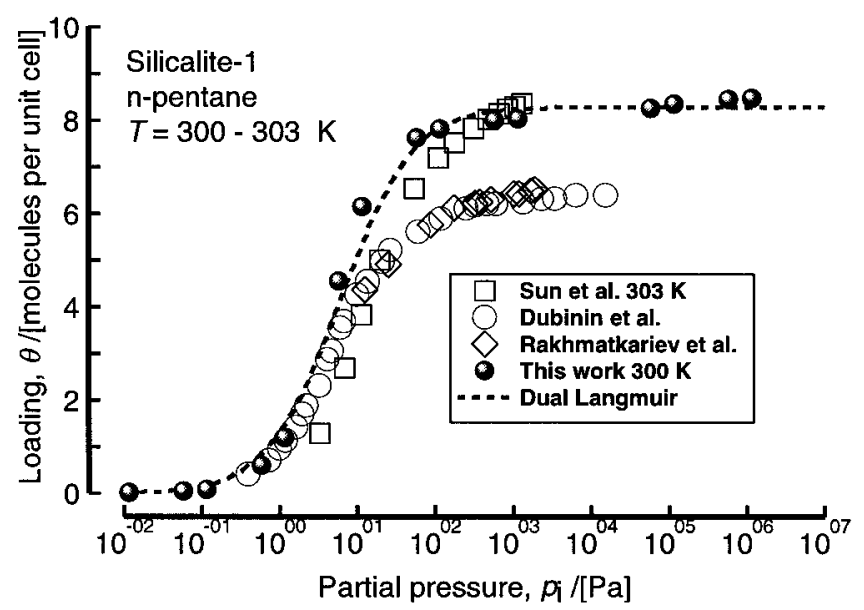

Figure 5. Comparison of adsorption isotherms of pentane in silicalite.

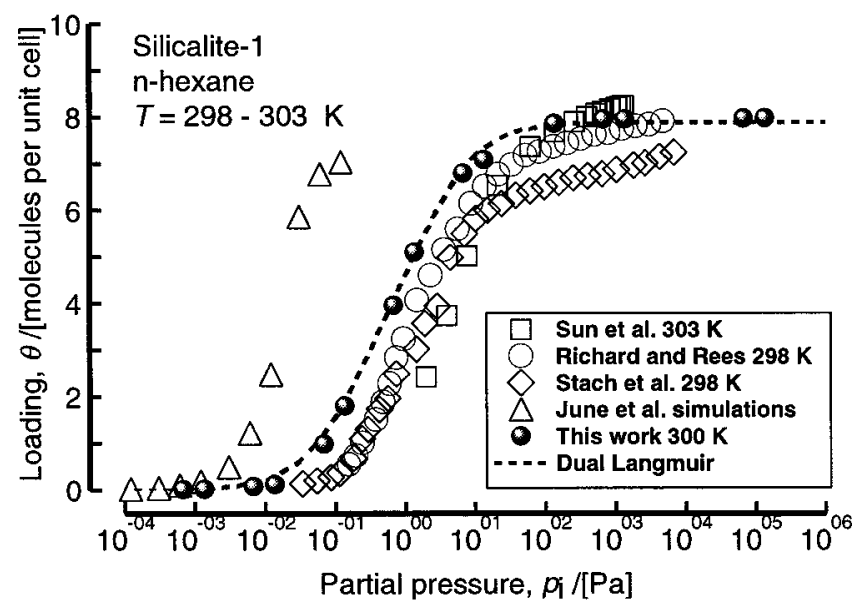

Figure 6. Comparison of adsorption isotherms of hexane in silicalite.

model developed in this work. From the comparison with the Henry coefficients (see Figure 3 ) it was already clear that the model of June et al. would overestimate the adsorption significantly. Our model gives a better agreement with experiments.

For heptane, adsorption isotherms have been reported by Lohse and Fahlke, ${ }^{3}$ Rakhmatkariev et al. ${ }^{4}$ Dubinin et al. ${ }^{5}$ and Sun et al. ${ }^{9}$ The simulations agree very well with the data of Sun et al. Since Rakhmatkariev et al. and Dubinin et al. used the same zeolite as for the experiments of pentane a similar difference as observed for pentane has to be expected with their data and our simulation results (see Figure 7). Although the agreement with experimental data of Rakhmatkariev et al. and Dubinin et al. is less satisfactory, both sets of experimental data show an inflection at a loading of adsorbate loading of four molecules per unit cell. This inflection is also observed in the simulated adsorption isotherms. In the next section we will discuss this aspect in detail.

For octane and nonane, the simulation results are compared with the data of Sun et al. ${ }^{9}$ in Figures 8 and 9, respectively. It is interesting to note that our simulations show a pronounced inflection at a loading of four molecules per unit cell. The experimental data of Sun et al. were obtained above this loading and therefore no inflection was noted experimentally. The agreement between the simulation results and experiments would improve significantly if the model would yield three times larger Henry coefficients (see Figure 3). The precise reason for the inflection behavior of these molecules is as yet unclear to us. The experimental data of Yang and Rees ${ }^{11}$ indicate 


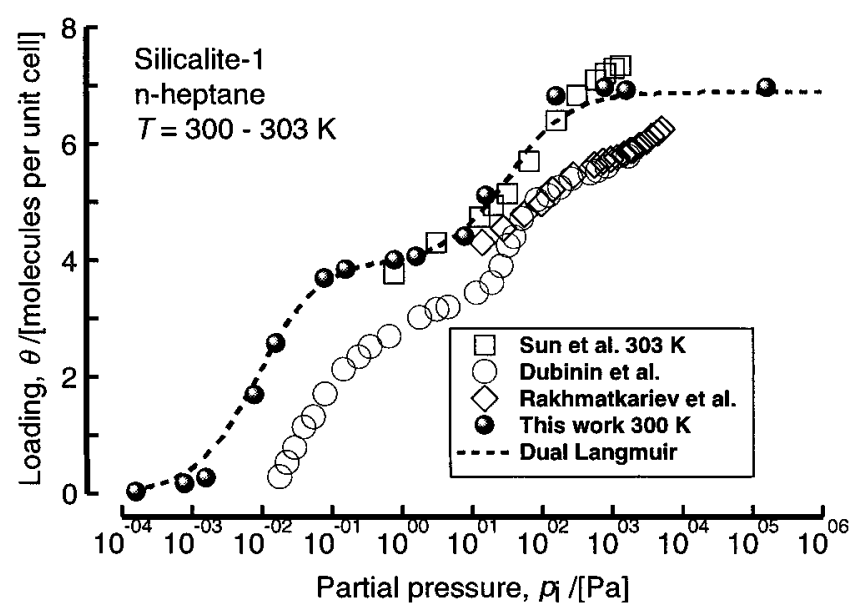

Figure 7. Comparison of adsorption isotherms of heptane in silicalite.

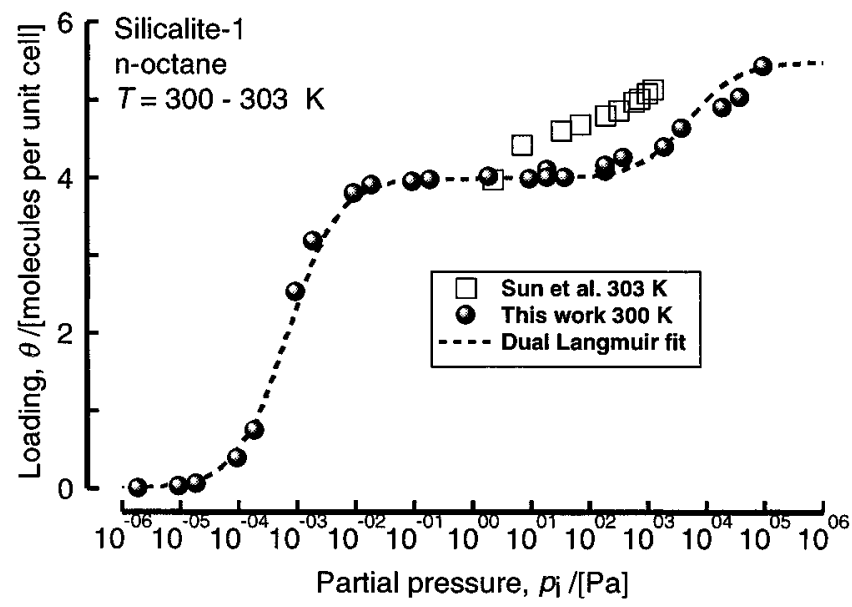

Figure 8. Comparison of adsorption isotherms of octane in silicalite.

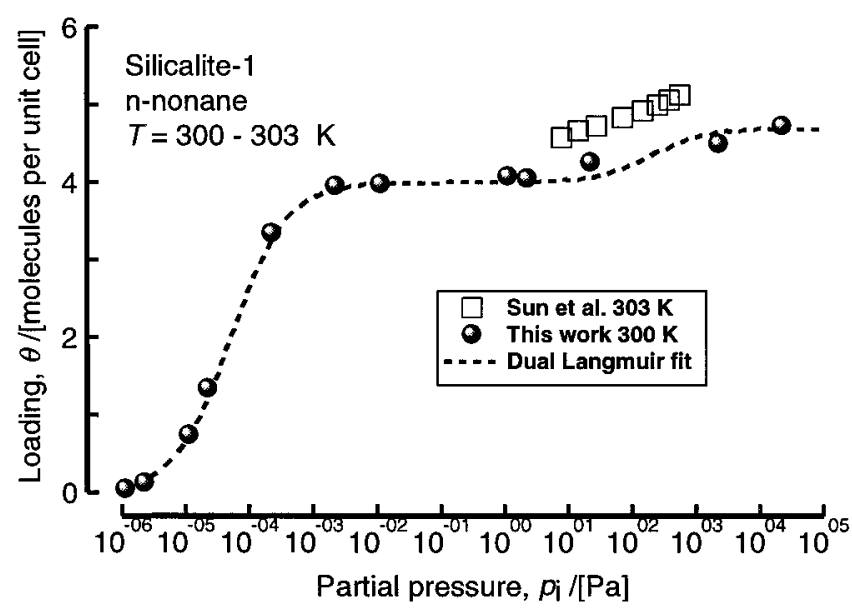

Figure 9. Comparison of adsorption isotherms of nonane in silicalite.

inflection behavior for octane and nonane. At this point it is important to note that the number of accepted exchanges with the reservoir in the CBMC scheme becomes for these molecules at high pressures (above $100 \mathrm{~Pa}$ ) very low. Therefore we had to increase the total length of the simulation and the total number of trial orientations significantly. We have performed simulations starting from a low loading and increasing the pressure as well as simulations starting from a high loading and decreasing the pressure. Both gave identical results. Therefore we do have some confidence that the inflection is not due to limitations of the CBMC technique. Furthermore, for these large molecules at

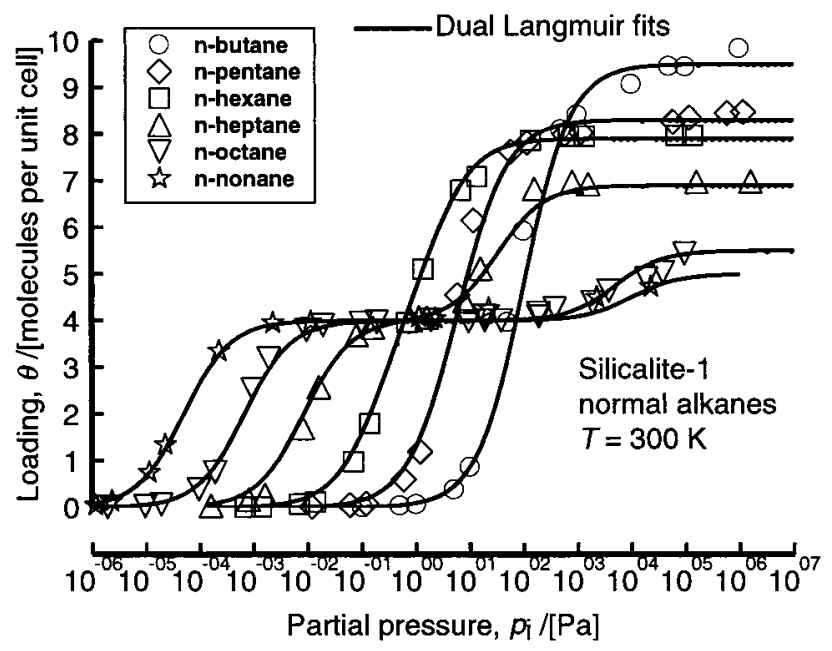

Figure 10. Simulated isotherms for $\mathrm{C}_{4}-\mathrm{C}_{9}$ linear alkanes in silicalite at $300 \mathrm{~K}$

these high pressures an important question is whether the assumption of the zeolite being rigid is still reasonable.

In Figure 10 the simulated isotherms for linear alkanes have been collected together for comparison and discussion. The continuous lines in Figure 10 are fits of the CBMC simulations using the dual-site Langmuir model, developed in Section 6.

C. Discussion. The adsorption isotherm of heptane shows a distinct inflection which suggests that a phase-transition takes places in the pores of the zeolite. A well-known example of a phase transition in porous systems is capillary condensation. If, in a system, capillary condensation is observed the adsorption isotherm shows a step and hysteresis occurs, such isotherms are denoted as type IV or V. ${ }^{51}$ Steps or inflections without hysteresis are occasionally observed in adsorption isotherms. Such adsorption isotherms are classified as type VI isotherms. These steps are usually due to wetting or preadsorption and occur mainly on flat surfaces. ${ }^{52}$ The pores of most zeolites are too small to observe capillary condensation. In these narrow pores the fluid behaves as a quasi one-dimensional fluid and in such a one-dimensional system phase transitions do not occur. ${ }^{55}$ Therefore for zeolites one would expect that for the linear alkanes the adsorption isotherms are of the type I. If a stepped adsorption isotherm is observed, this step is usually attributed to capillary condensation in the exterior secondary pore system formed by the space between the different crystals. ${ }^{2}$ If such a measurement would have been performed with a perfect crystal, an ordinary type I isotherm would have been observed. For linear alkanes with five or less carbon atoms a simple Langmuir isotherm has been found. ${ }^{56}$ Also temperature-programmed desorption studies show that among the linear alkanes hexane and heptane behave distinctly differently. ${ }^{7,8,10,12}$ Therefore the results for heptane and also hexane are surprising and in this section we discuss these results in detail.

Detailed inspection of the hexane experimental data of Richard and $\operatorname{Rees}^{6}$ suggests that a small kink is present at about four molecules per unit cell at $T=333 \mathrm{~K}$. In addition, the data in ref 6 indicates that with increasing temperature this inflection becomes more pronounced. Stach et al. ${ }^{2}$ and Lohse et al. ${ }^{57}$ did not observe an inflection at room temperature. Eder and Lercher ${ }^{58-61}$ observed an inflection at $T=333 \mathrm{~K}$. Yang and Rees ${ }^{10}$ also observed that this inflection disappears when the temperature is increased above $T=383 \mathrm{~K}$. Sun et al. ${ }^{9}$ state that an inflection is observed in a narrow temperature window $(310<T<360 \mathrm{~K})$, below and above this temperature 


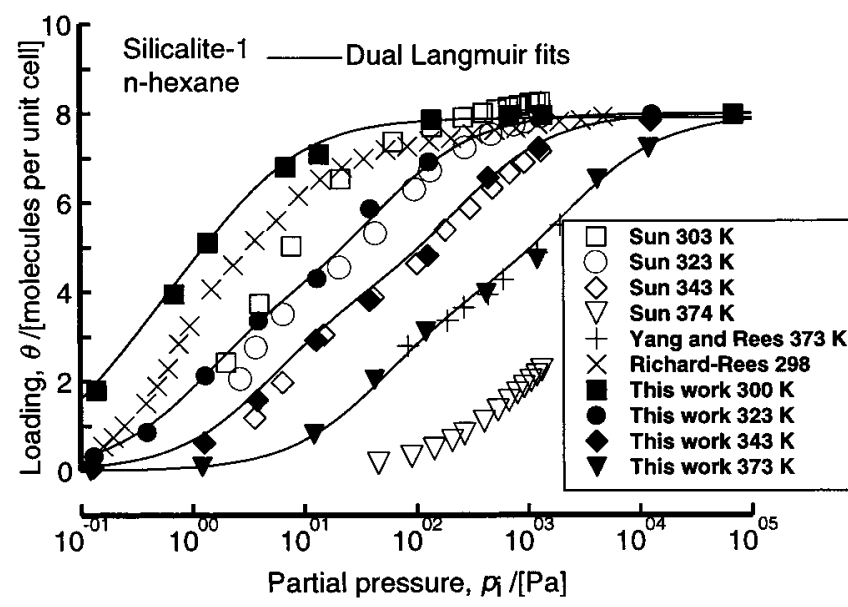

Figure 11. Adsorption isotherms of hexane in silicalite at various temperatures.

window normal type I isotherms are observed. For heptane both the experiments and simulations show a pronounced inflection.

The anomalous behavior of hexane and heptane in silicalite compared to other alkanes is now well established. However, the temperature dependence of the inflection of hexane and heptane is still debated in the literature. The simulation results for hexane of Smit and Maesen ${ }^{13}$ indicate that as the temperature increases the inflection becomes more pronounced. The experimental data of Richard and Rees ${ }^{6}$ appear to support this point. However, recently Sun et al. ${ }^{9}$ and Yang and Rees ${ }^{10}$ claim that their experimental data indicate that as the temperature is increased the inflection disappears. It is therefore interesting to investigate the temperature dependence of the inflection in detail. In Figure 11 the simulated adsorption isotherms of hexane obtained at temperatures ranging from 298 to $373 \mathrm{~K}$ are compared with the experimental data of Sun et al., ${ }^{9}$ Richard and Rees, ${ }^{6}$ and Yang and Rees. ${ }^{10}$ At about $300 \mathrm{~K}$ the simulations are in good agreement with the data of Richard and Rees ${ }^{6}$ but deviate slightly from the data of Sun et al. There is excellent agreement between the simulations at 323 and $343 \mathrm{~K}$ and the experimental data of Sun et al. ${ }^{9}$ When the temperature is further increased to $373 \mathrm{~K}$, we note that the experimental data of Sun et al. are significantly below the simulation results. The reason for this deviation is unclear. It is important to note that our simulations at $373 \mathrm{~K}$ are in excellent agreement with the data of Yang and Rees. ${ }^{10}$ Our simulations show a regular shift of the isotherm toward higher pressures if the temperature is increased; this agrees with the experimental observations of Yang and Rees, ${ }^{10}$ but not with those of Sun et al.

At room temperatures both the experiments and the simulations show an inflection at a loading of four molecules per unit cell. At high temperatures all simulated adsorption isotherms show inflection behavior. Simulation at $T=1000 \mathrm{~K}$ have confirmed this. A careful examination of our simulation results and also the experimental data of Yang and Rees ${ }^{10}$ shows that these results are in very good agreement. It also shows that from the experimental data it is difficult to conclude whether an inflection is present at higher temperature. Our CBMC simulations do not support the contention of Yang and Rees that the inflection behavior disappears at higher temperatures. As is shown in Figure 11, the isotherm data of Sun et al. at high temperatures were not obtained at sufficiently high pressures in order to notice inflection behavior. Therefore, the observation of Sun et al. that the inflection behavior of $n$-hexane is restricted to a temperature window $(310<T<360 \mathrm{~K})$ is also not borne out. Figure 12 compares the experimental adsorption isotherms

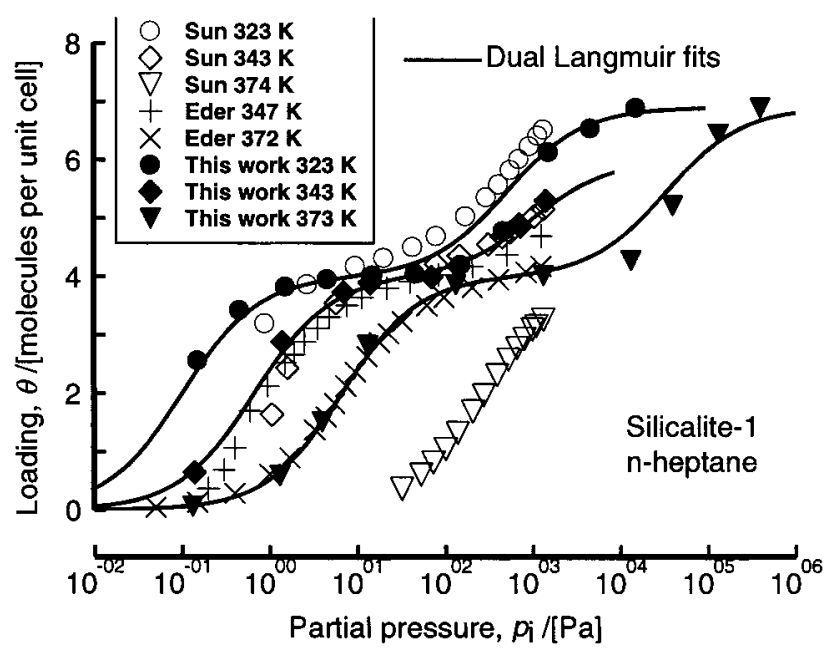

Figure 12. Adsorption isotherms of heptane in silicalite at various temperatures.

of heptane of Sun et al. ${ }^{9}$ and Eder ${ }^{58,60-62}$ obtained at temperatures of 323,343 , and $373 \mathrm{~K}$ with the simulation results. At $323 \mathrm{~K}$ the simulations are in good agreement with the data of Sun et al. There is excellent agreement between the simulations at $T=343$ with the experiments of both Sun et al. and Eder. At $T=373 \mathrm{~K}$ the CBMC simulations agree very well with the data of Eder but there is a significant deviation from the Sun et al. data. This deviation is similar to the one observed earlier for hexane at $T=373$ (see Figure 11). The inflection for heptane is found by Rakhmatkariev et al. ${ }^{4}$ and Dubinin et al. ${ }^{5}$ at room temperature and at slightly higher temperatures by Eder and Lercher $^{58-61}$ and Sun et al. ${ }^{9}$ As is shown in Figure 12, the isotherm data of Sun et al. at high temperatures were not obtained at sufficiently high pressures in order to notice inflection behavior. Therefore, the conclusion of Sun et al. that the inflection behavior of $n$-heptane occurs in a temperature window is not supported by our results. In the case of heptane the results clearly show that with increasing temperature the inflection behavior becomes more pronounced.

A possible explanation of the peculiar behavior of heptane and hexane was given by Smit and Maesen ${ }^{13}$ in terms of a commensurate freezing of hexane and heptane in the zigzag channels of silicalite. Only hexane and heptane have a size that is commensurate with the size of the zigzag channel. This effect is illustrated in Figure 13 in which we compare the density distribution of the center of mass of hexane at low pressure and high pressure. At low pressure we observe a uniform distribution of the molecules in the intersections, straight and zigzag channels. This distribution completely changes at high pressures where the molecules are localized into the zigzag channels in such a way that the intersections are free. This allows a complete filling of the straight channels, in which we observe a nearly uniform distribution. It is interesting to compare this distribution of hexane with the distribution of pentane and butane at high loadings (see Figure 14). For butane we observe a nearly uniform distribution. For pentane this distribution is less uniform, but the dots are not as clearly clustered as for hexane indicating that the strong localization in the zigzag channels is not present.

Another evidence that the packing efficiency of hexane and heptane are higher than that of other linear alkanes can be obtained by plotting the maximum loading expressed in $\mathrm{kg}$ per $\mathrm{kg}$ of silicalite against the number of carbon atoms (see Figure $15)$; there is a clear maximum loading for hexane and heptane. 
(a)

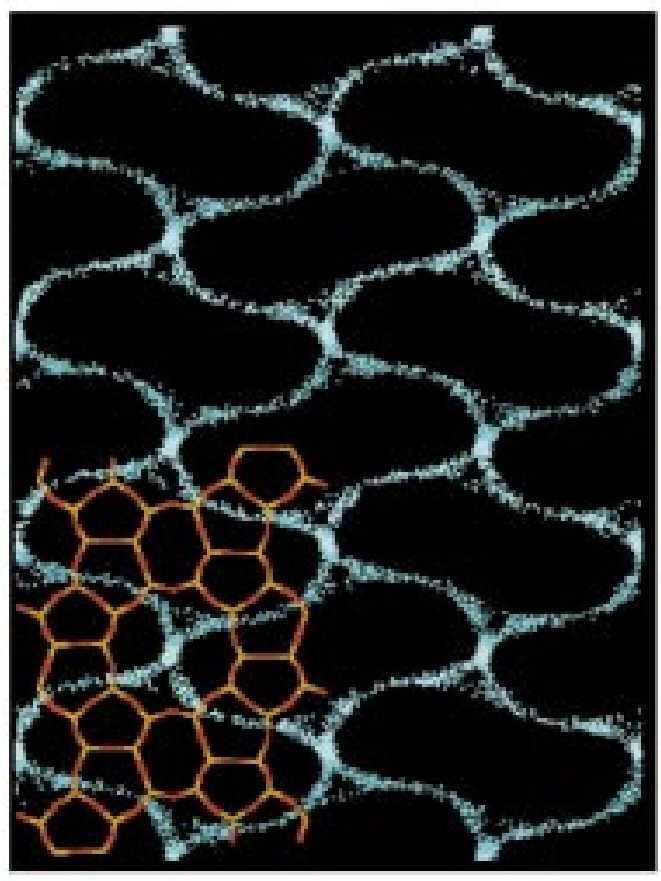

(c)

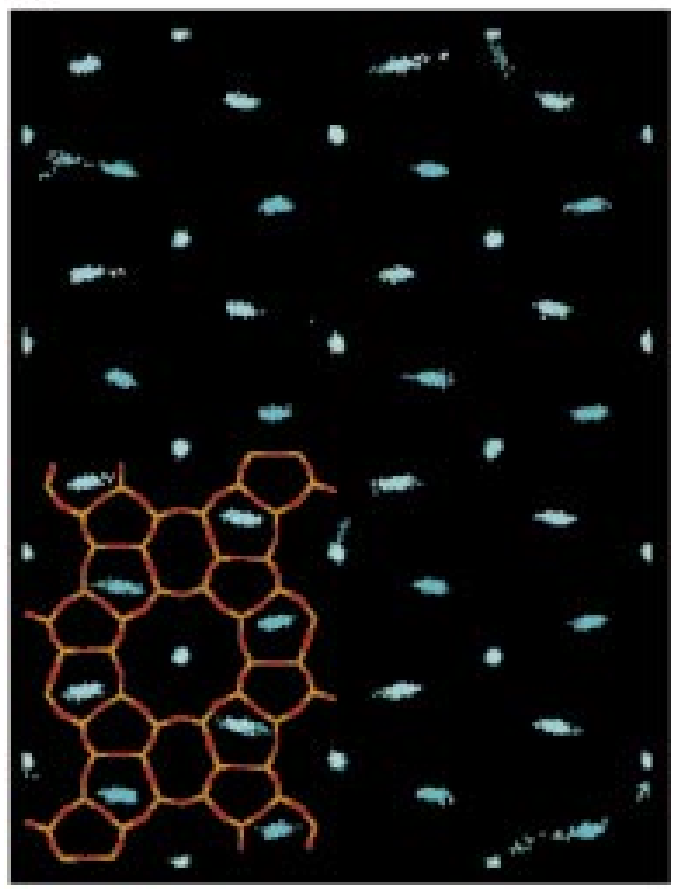

(b)

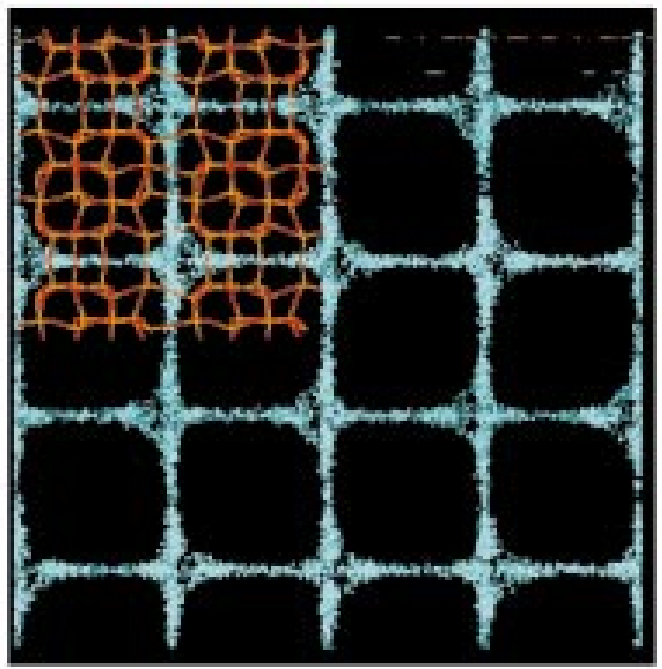

(d)

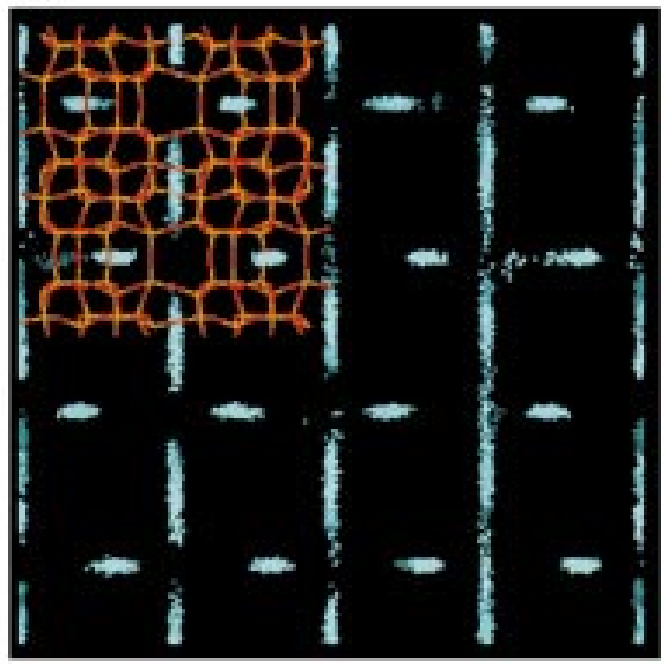

Figure 13. Probability distribution of hexane in silicalite at $T=405 \mathrm{~K}$ : (left figures) projection on the $x-z$ plane, (right figures) projection on the $x-y$ plane; low pressures $0.01 \mathrm{kPa}$ (top figures) and high pressures $1000 \mathrm{kPa}$ (bottom figures). At intervals of 100 cycles the center of mass of a hexane molecule is computed and at this position a dot is drawn; this is repeated until 10000 dots have been plotted. The lines are the zeolite structure (only a quarter of the total zeolite used in the simulation is shown).

Expressed in terms of molecules per unit cell, the maximum loading decreases with increasing carbon number in a monotonic fashion.

\section{Branched Alkanes}

Compared to linear alkanes much less experimental data is available on the adsorption of branched alkanes in silicalite. Adsorption isotherms of isobutane have been reported by Sun et al. ${ }^{16}$ and Zhu et al. ${ }^{14}$ for various hexane isomers by Cavalcante and Ruthven, ${ }^{17}$ and for 2-methylheptane by Eder. ${ }^{58,60,61}$ Simulations of branched alkanes have been reported in refs 63, 64. June et al. showed that at infinite dilution the branched alkanes prefer the intersections. These observations were confirmed by the simulations of Smit and co-workers. ${ }^{15,64}$ Here we investigate the sorption behavior of branched alkanes at higher loadings. As a first approximation we have assumed that the interaction $\mathrm{CH}$ group of the branched alkane with the zeolite is identical to the interaction of a $\mathrm{CH}_{2}$ group (see Table 2). Experimentally 
(a)

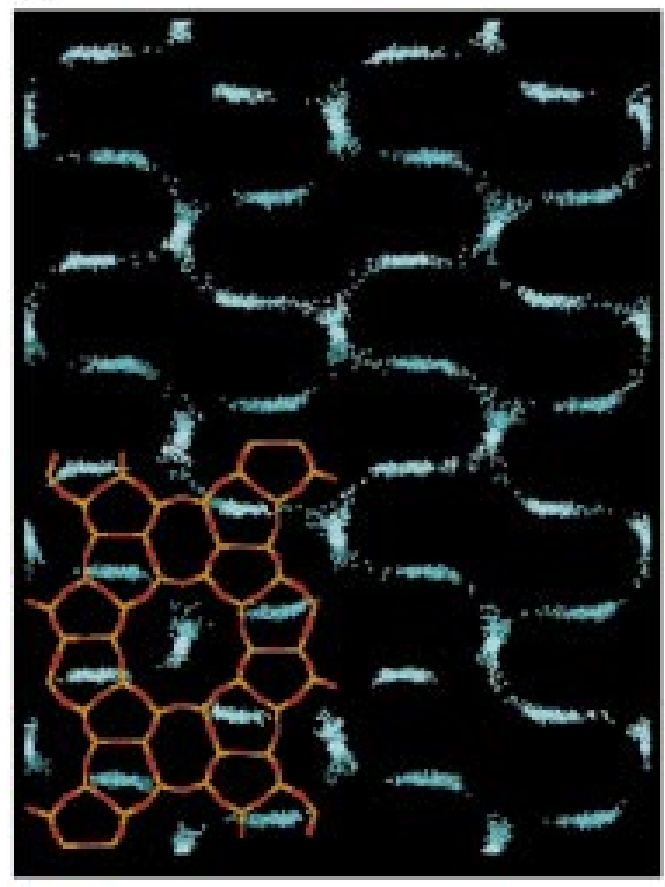

(c)

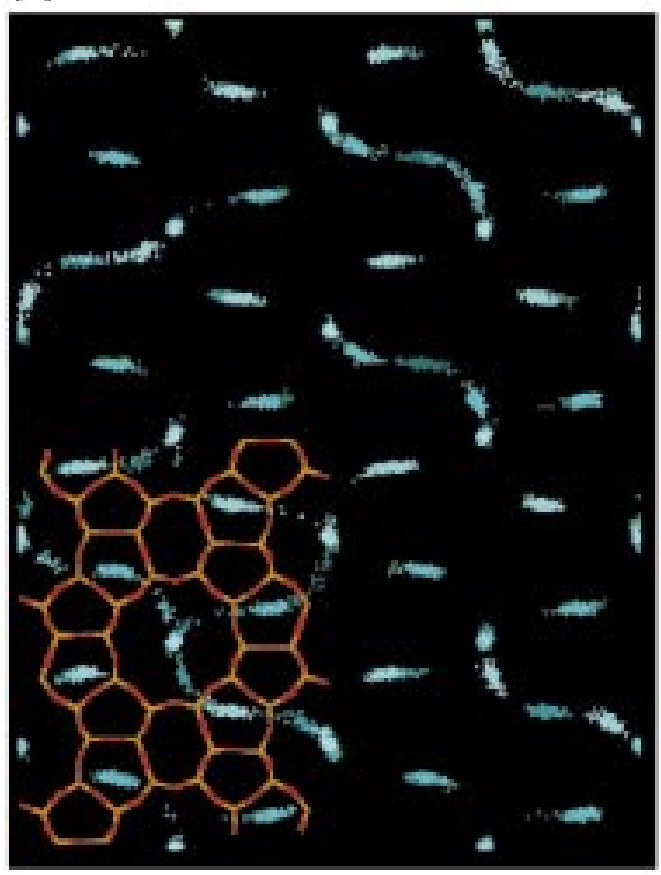

(b)

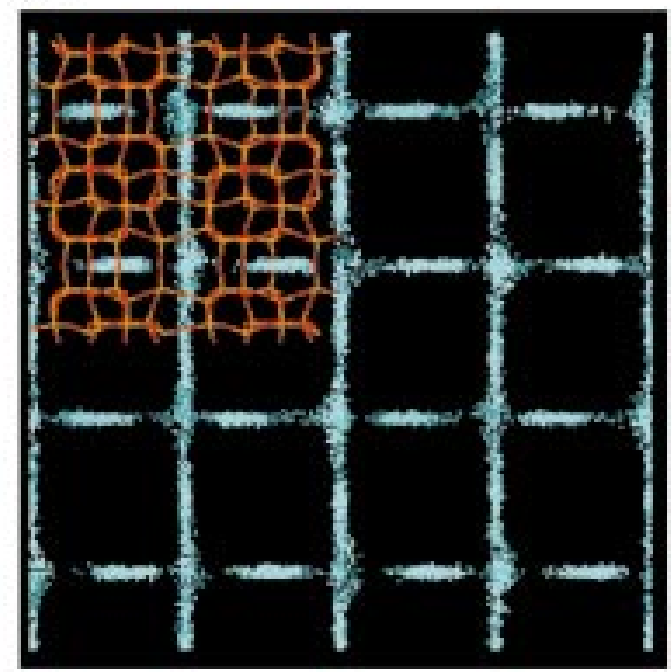

(d)

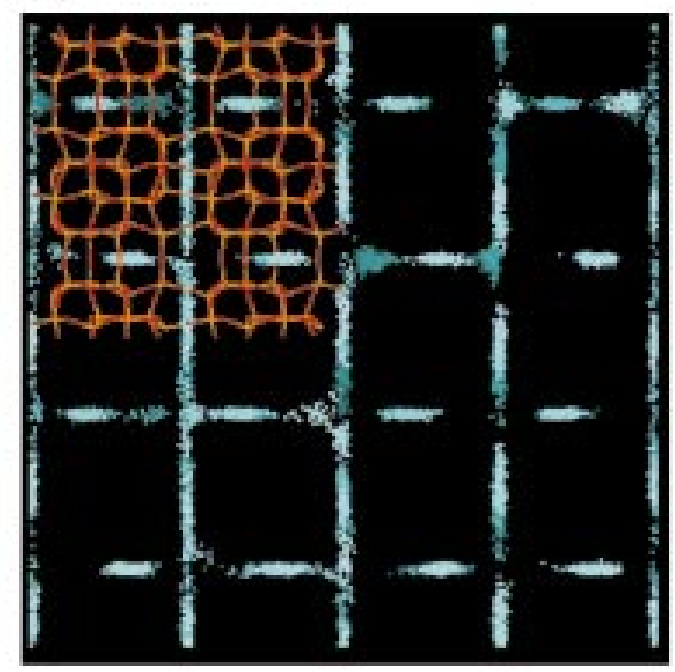

Figure 14. Probability distribution of butane (top figures) and pentane (bottom figures) in silicalite at $T=300 \mathrm{~K}$ : (left figures) projection on the $x-z$ plane projection, (right figures) projection on the $x-y$ plane at high pressures $100 \mathrm{kPa}$. At intervals of 400 cycles the center of mass of a molecule is computed and at this position a dot is drawn; this is repeated until 10000 dots have been plotted.

the heats of adsorption of isobutane have been obtained by Zhu et al. ${ }^{14}$ and Sun et al. ${ }^{16}$ who obtained -46.7 and $-56 \mathrm{~kJ} /$ mol, respectively. The reasons for this large difference are unclear. For 2-methylpentane Cavalcante and Ruthven ${ }^{17}$ obtained $-68 \mathrm{~kJ} / \mathrm{mol}$, and Eder and Lercher ${ }^{58-61}$ report for 2-methylpentane a heat of adsorption of $-90 \mathrm{~kJ} / \mathrm{mol}$. Figure 16 shows that for the 2-methylalkanes our model gives very good results. For isobutane our simulations are in good agreement with the data of Zhu et al. but deviate significantly from the data of Sun et al.
In Figure 17 the simulated adsorption isotherm of isobutane is compared with the experimental isotherms of Sun et al. ${ }^{16}$ and Zhu et al.. ${ }^{14}$ The agreement is very good. Both the experiments and the simulations show an inflection at a loading of four molecules per unit cell. We had shown earlier that this inflection is due to a preferential adsorption of isobutane at the intersection. ${ }^{45}$ Only at high pressures additional molecules can adsorb in the straight and zigzag channels.

The simulated isotherms for 2-methylalkanes at $300 \mathrm{~K}$ temperature are shown in Figure 18. The continuous lines in 


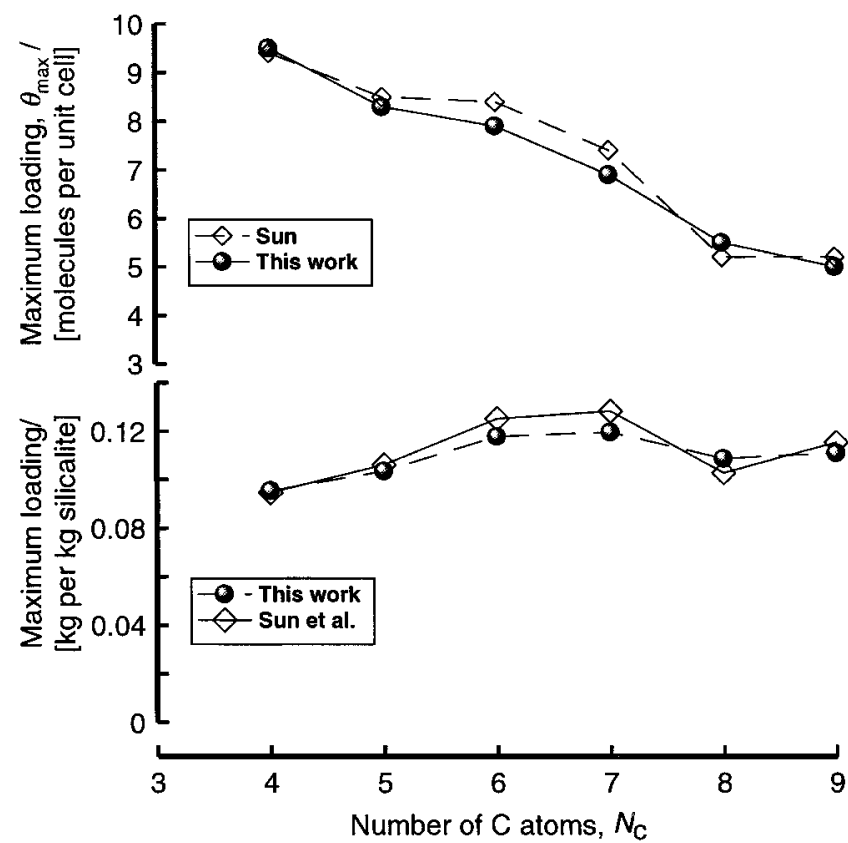

Figure 15. Comparison of the maximum loading for linear alkanes obtained from simulations with experimental data of Sun et al. ${ }^{16}$

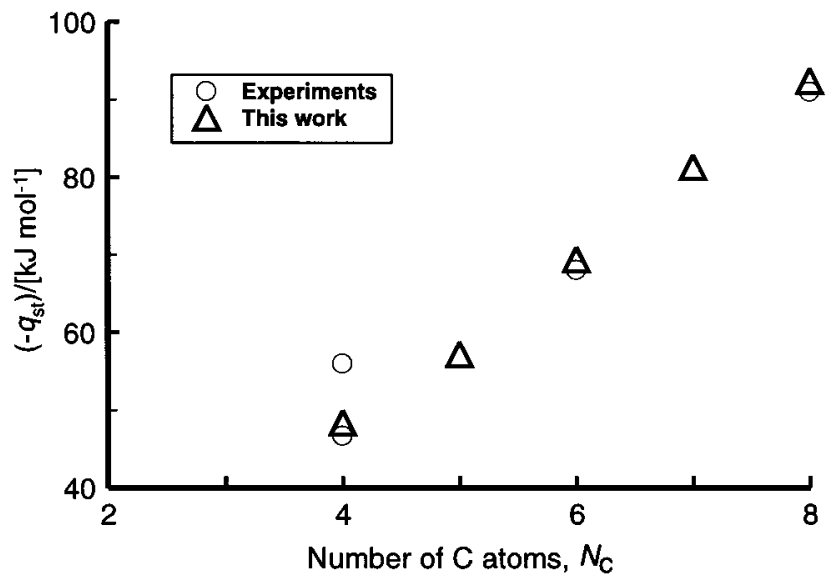

Figure 16. Heats of adsorption $\left(-q_{\mathrm{st}}\right)$ as a function of the number of carbon atoms $N_{\mathrm{c}}$ of the branched 2-methylalkanes adsorbed in silicalite.

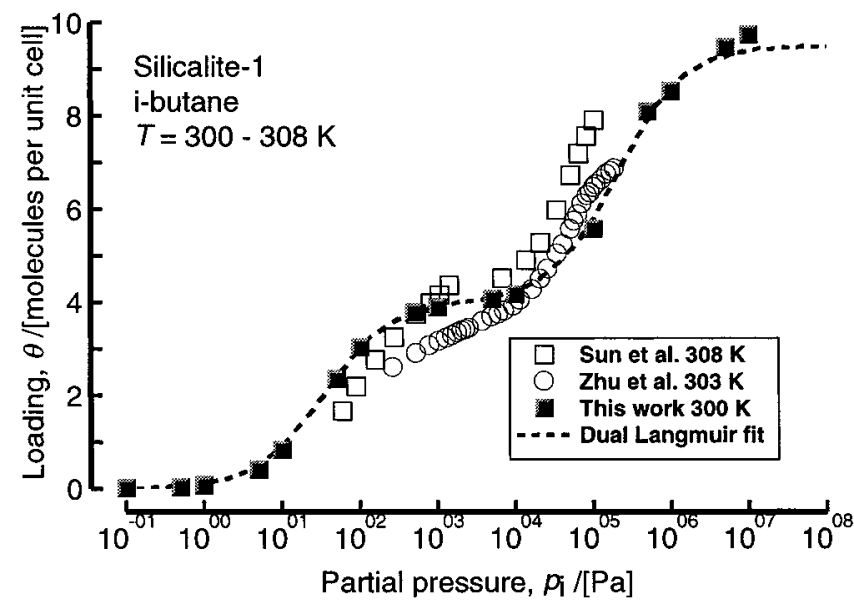

Figure 17. Comparison of adsorption isotherms of isobutane in silicalite.

this figure are fits of the CBMC simulations using the dual-site Langmuir model, developed in Section 6.

All branched hydrocarbons show inflection at a loading of four molecules per unit cell; this inflection is more pronounced

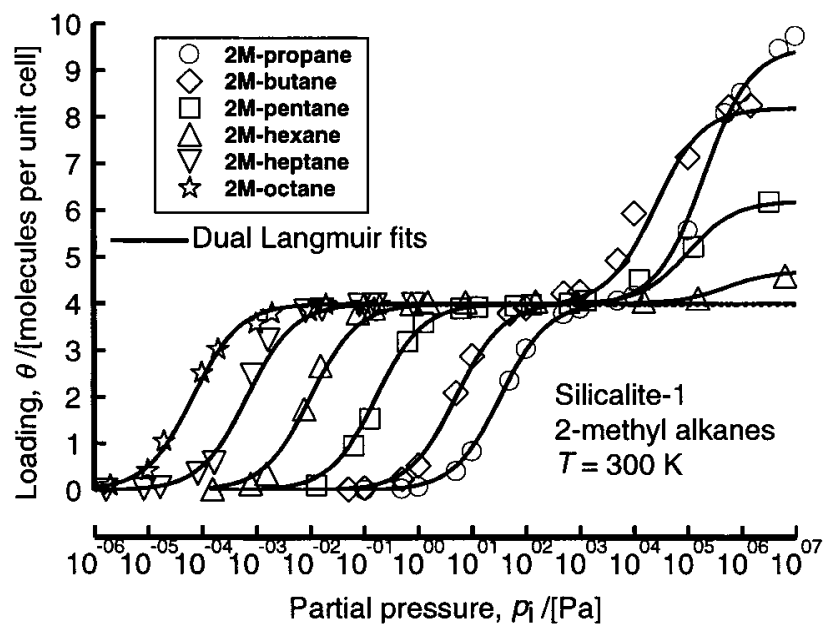

Figure 18. Simulated isotherms for branched alkanes in silicalite at $300 \mathrm{~K}$.

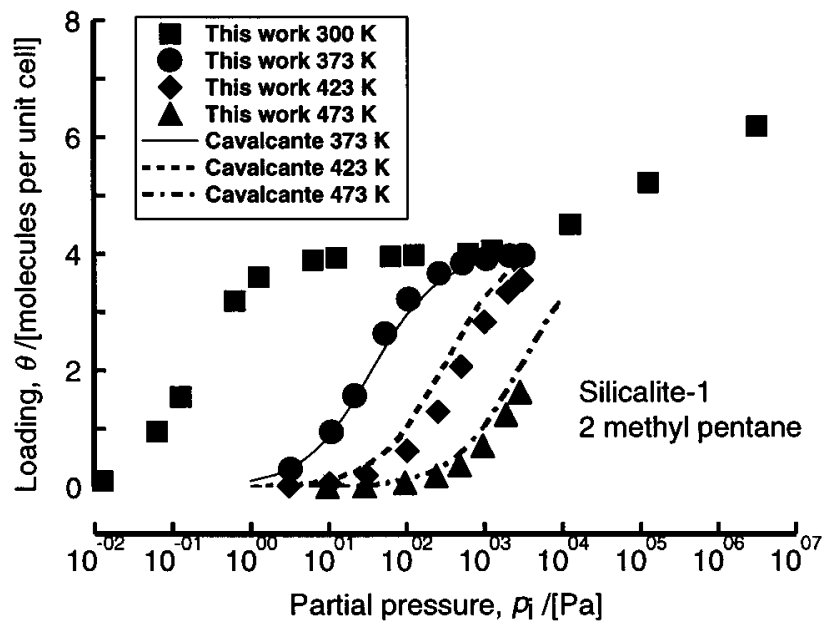

Figure 19. Adsorption isotherms of 2-methylpentane in silicalite at various temperatures.

as the chain length increases. For the longer branched alkanes adsorption isotherms have been measured by Cavalcante and Ruthven ${ }^{17}$ for 2-methylpentane, and by Eder and Lercher for 2-methylheptane. ${ }^{58,60,61}$ Interestingly these experimental adsorption isotherms do not show any inflection behavior. It is therefore necessary to investigate these apparent discrepancy in the observations regarding inflection.

In Figure 19 we compare the experimental data ${ }^{17}$ with our simulation results for 2-methylpentane. Considering the fact that we have optimized our parameters for linear alkanes using experimental data at room temperature, the agreement at these elevated temperatures is surprisingly good. This figure also makes clear that the pressures in the experiments were not sufficiently high to observe an inflection. Similar agreement between the experiments of Eder ${ }^{58,60,61}$ for 2-methylheptane at $T=372 \mathrm{~K}$ and our simulations are observed (see Figure 20). For the range of pressures studied both simulations and experiments do not exhibit an inflection behavior.

Figure 18 shows that the inflection becomes more pronounced as the chain length increases. The reason for this becomes clear if we investigate the siting of the molecules. In Figure 21 we compute the probability distribution of 2-methylhexane to have its tail in the zigzag (cyan dots) or straight channels (pink dots). At low pressures we observe that all molecules are located in the intersections and have an equal probability of having their tails in either the straight or zigzag channels. Interestingly, at 


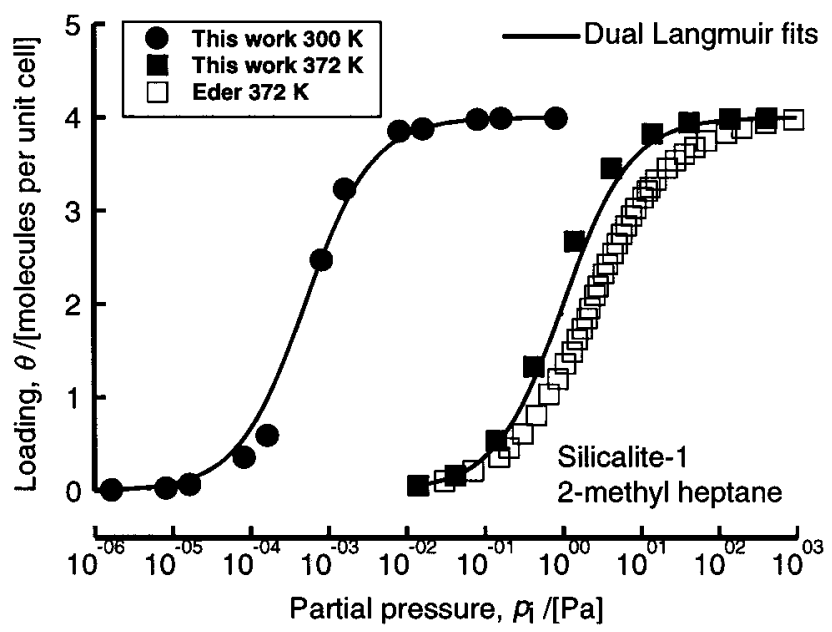

Figure 20. Adsorption isotherms of 2-methylheptane in silicalite.

high pressures this picture changes, molecules also adsorb between intersections. Figure 21 shows that this occurs only in the zigzag channels, additional molecules do not adsorb in the straight channels. This has consequences for the orientation of the tails. To evacuate the zigzag channels there is a collective reorientation of the tails such that most tails are in the straight channels. Such a collective reorientation is not required for isobutane and may explain the more pronounced inflection when the chain length is increased beyond four carbon atoms.

\section{Fitting of Simulated Isotherms with the Dual-Site Langmuir Model}

The isotherm inflection behavior observed for branched alkanes (see Figure 18) and for linear alkanes with six or more $\mathrm{C}$ atoms (see Figure 10) cannot be modeled using a simple Langmuir isotherm. Sun et al.9,16 have used a six-parameter Virial-type equation to fit these isotherms. In this section, we develop a much simpler approach based on the molecular insight obtained from the simulations. From the discussion regarding the inflection behavior of isobutane it becomes clear that one must account for differences in the ease with which a molecule can be adsorbed at the intersections and within the channel interiors. We therefore adopt a dual-site Langmuir model ${ }^{65,66}$ for purposes of fitting the isotherms

$$
\begin{aligned}
& \theta=\frac{\theta_{\mathrm{A}} k_{\mathrm{A}} p}{1+k_{\mathrm{A}} p}+\frac{\theta_{\mathrm{B}} k_{\mathrm{B}} p}{1+k_{\mathrm{B}} p}= \\
& \frac{\left(\theta_{\mathrm{A}} k_{\mathrm{A}}+\theta_{\mathrm{B}} k_{\mathrm{B}}\right) p+\left(\theta_{\mathrm{A}}+\theta_{\mathrm{B}}\right) k_{\mathrm{A}} k_{\mathrm{B}} p^{2}}{1+\left(k_{\mathrm{A}}+k_{\mathrm{B}}\right) p+k_{\mathrm{A}} k_{\mathrm{B}} p^{2}}
\end{aligned}
$$

where we identify sites $\mathrm{A}$ and $\mathrm{B}$, with the respective maximum loading capacities $\theta_{\mathrm{A}}$ and $\theta_{\mathrm{B}}$, expressed in molecules per unit cell, $p$ is the partial pressure of the component. The dual-site Langmuir constants for adsorption at the two sites A and B are $k_{\mathrm{A}}$ and $k_{\mathrm{B}}$ (expressed in $\mathrm{Pa}^{1-}$ ). We take site $\mathrm{A}$ to be the one with the higher Langmuir constant. From Figures 10 and 18, it is clear that inflection in silicalite occurs at a loading of four molecules per unit cell and so the maximum capacity of site A, so $\theta_{\mathrm{A}}=4$. From Figures 10 and 18 we conclude that $\theta_{\mathrm{A}}$ should be taken equal to 4 for all (linear and branched) alkanes $\left(\theta_{\mathrm{A}}\right.$ is therefore not used as a fitting parameter). The maximum total loading $\theta_{\max }=\theta_{\mathrm{A}}+\theta_{\mathrm{B}}$ for the linear alkanes from the simulations agree with the experimental data of Sun et al. ${ }^{67}$ (see Figure 15). In our description of the data we have used the values of $\theta_{\max }$ corresponding to our simulation results; this is therefore also not a fit parameter. All our CBMC results shown in Figures 10 and 18 were described by fitting the two remaining Langmuir constants $k_{\mathrm{A}}$ and $k_{\mathrm{B}}$ to eq 1 . The fitted curves describe the simulation results exceedingly well; see Figure 10, 18, and also other figures presented here. The values of the fit parameters for linear and branched alkanes are presented in Figure 22 in the form

$$
k=\frac{\left(\theta_{\mathrm{A}} k_{\mathrm{B}}+\theta_{\mathrm{B}} k_{\mathrm{B}}\right)}{\theta_{\mathrm{A}}} ; S=k_{\mathrm{A}} k_{\mathrm{B}}
$$

The fitted parameter $k$ is practically identical for linear and branched alkanes. The $S$ parameter, on the other hand, is about $2-3$ orders of magnitude lower for the branched alkanes as compared to the linear ones. This causes the inflection behavior for branched alkanes to be much more prominent. The information presented in Figure 22 could be extrapolated to estimate the isotherms for alkanes with higher carbon numbers. We note in passing that the constant $k \times \theta_{\max }$ presented in Figure 19 corresponds remarkably well with the Henry coefficients shown in Figure 3.

There is an important advantage in being able to describe the inflection behavior accurately with the help of the dual-site Langmuir model; this is because it would then be possible to predict the mixture isotherm from only pure component data. There are two ways to set up the mixture rule. In the first approach (I) we apply this rule to each of the two sites A and B separately. For each site we apply the multicomponent extension of the Langmuir isotherm; ${ }^{66}$ for a mixture of components 1 and 2, therefore, this rule yields:

$$
\begin{aligned}
& \theta_{1}=\frac{\theta_{\mathrm{A} 1} k_{\mathrm{A} 1} p_{1}}{1+k_{\mathrm{A} 1} p_{1}+k_{\mathrm{A} 2} p_{2}}+\frac{\theta_{\mathrm{B} 1} k_{\mathrm{B} 1} p_{1}}{1+k_{\mathrm{B} 1} p_{1}+k_{\mathrm{B} 2} p_{2}} \\
& \theta_{2}=\frac{\theta_{\mathrm{A} 2} k_{\mathrm{A} 2} p_{2}}{1+k_{\mathrm{A} 1} p_{1}+k_{\mathrm{A} 2} p_{2}}+\frac{\theta_{\mathrm{B} 2} k_{\mathrm{B} 2} p_{2}}{1+k_{\mathrm{B} 1} p_{1}+k_{\mathrm{B} 2} p_{2}}
\end{aligned}
$$

where $k_{\mathrm{A} i}$ and $k_{\mathrm{B} i}$ are the Langmuir constants for species $i$ for sites $\mathrm{A}$ and $\mathrm{B}$, and $p_{i}$ is the partial pressure of the component $i$ in the gas phase. We expect this mixture scenario to hold when each of the two components 1 and 2 is present in both sites.

The second scenario (II) is to apply the mixture rule to the combination of sites $(\mathrm{A}+\mathrm{B})$. This scenario is appropriate to situations in which one of the components is excluded from one particular site (say B) due to a higher (free) energy barrier; therefore we set up the mixing rule for the total of $(A+B)$, i.e., the entire zeolite. To derive this mixing rule, the most convenient starting point is the right equality of eq 1 and the guidelines outlined in the book of Ruthven. ${ }^{66}$ This yields for a two-component system the following set of equations:

$$
\begin{aligned}
\theta_{1} & =\frac{\left(\theta_{\mathrm{A} 1} k_{\mathrm{A} 1}+\theta_{\mathrm{B} 1} k_{\mathrm{B} 1}\right) p_{1}+\left(\theta_{\mathrm{A} 1}+\theta_{\mathrm{B} 1}\right) k_{\mathrm{A} 1} k_{\mathrm{B} 1} p_{1}^{2}}{1+\left(k_{\mathrm{A} 1}+k_{\mathrm{B} 1}\right) p_{1}+k_{\mathrm{A} 1} k_{\mathrm{B} 1} p_{1}^{2}+\left(k_{\mathrm{A} 2}+k_{\mathrm{B} 2}\right) p_{2}+k_{\mathrm{A} 2} k_{\mathrm{B} 2} p_{2}^{2}} \\
\theta_{2} & = \\
& \frac{\left(\theta_{\mathrm{A} 2} k_{\mathrm{A} 2}+\theta_{\mathrm{B} 2} k_{\mathrm{B} 2}\right) p_{2}+\left(\theta_{\mathrm{A} 2}+\theta_{\mathrm{B} 2}\right) k_{\mathrm{A} 2} k_{\mathrm{B} 2} p_{2}^{2}}{1+\left(k_{\mathrm{A} 1}+k_{\mathrm{B} 1}\right) p_{1}+k_{\mathrm{A} 1} k_{\mathrm{B} 1} p_{1}^{2}+\left(k_{\mathrm{A} 2}+k_{\mathrm{B} 2}\right) p_{2}+k_{\mathrm{A} 2} k_{\mathrm{B} 2} p_{2}^{2}}
\end{aligned}
$$

In the following section we compare the predictions of the two mixture rules, based on pure component data with simulation results. 
(a)

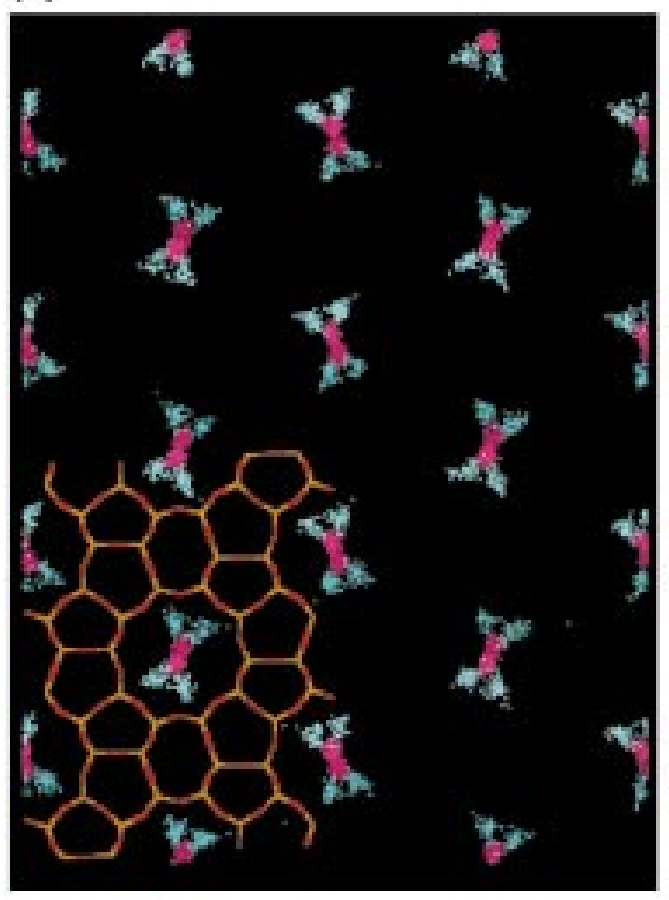

(c)

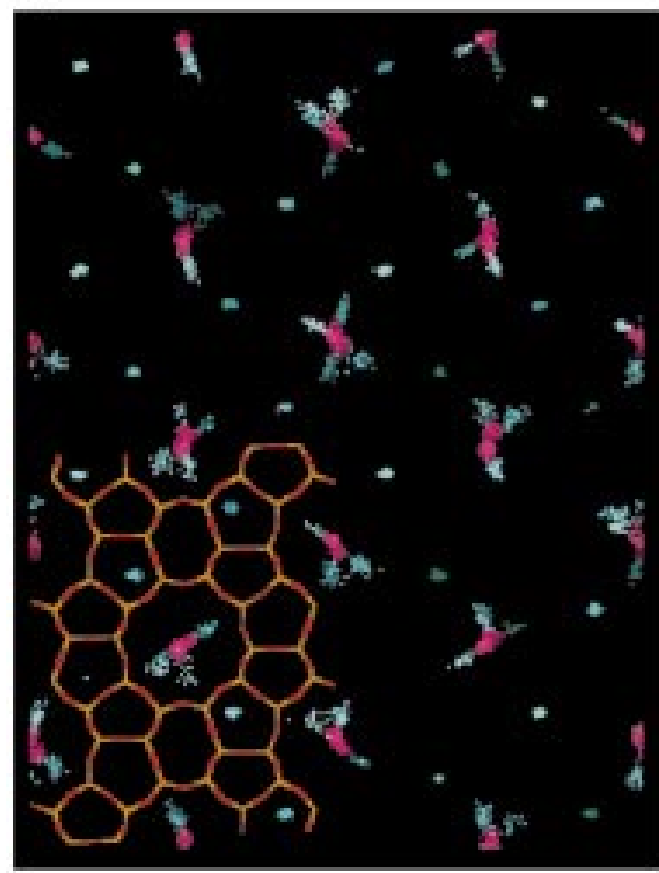

(b)

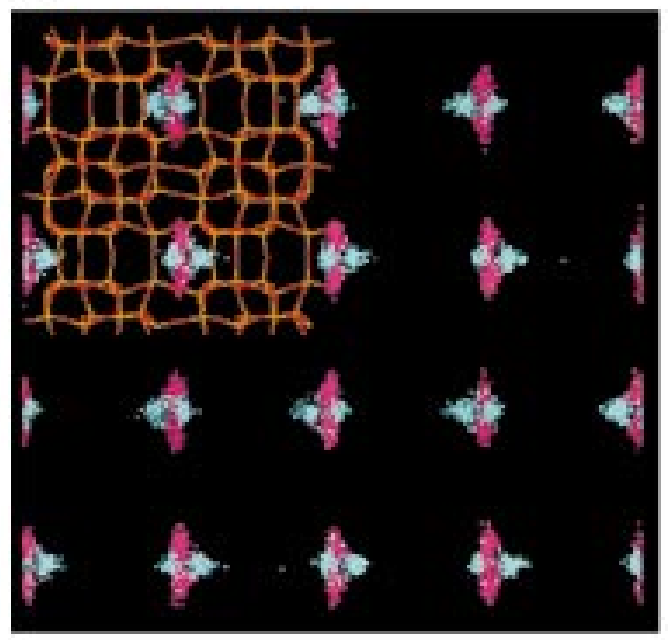

(d)

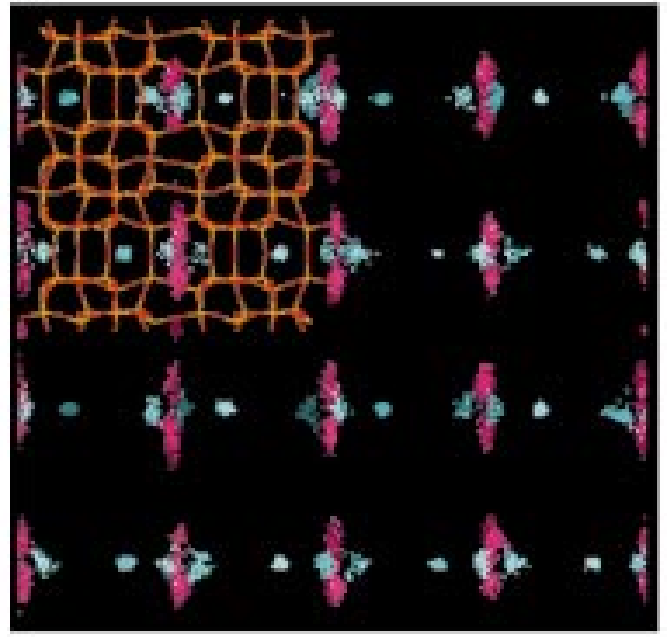

Figure 21. Probability distribution of the orientation of the tails of 2-methylpentane at various pressures at $T=300 \mathrm{~K}$. Every 350 th Monte Carlo cycle the center of mass of a molecule is computed and a cyan dot is drawn if the molecule has its tail in one of the zigzag channels or a pink dot if the tail resides in the straight channel. The total number of points is 8000 . The top figures are for $p=0.01 \mathrm{kPa}$, and the bottom figures are for $500 \mathrm{kPa}$. The left Figures are projections on the top plane, and the right figures are projections on the front plane.

\section{Mixtures}

In the previous sections we have shown that our model gives a satisfactory description of the adsorption isotherms of $n$ alkanes and 2-methylalkanes for $\mathrm{C}_{4}-\mathrm{C}_{9}$. In this section we investigate the mixture isotherms of various alkane isomers.

Experimentally, the measurement of an isotherm is more complicated for mixtures than for pure components. One has to measure not only the weight increase of the zeolite as a function of pressure but also the change in composition of the gas mixture. To the best of our knowledge, only adsorption isotherms of mixtures of short alkanes have been measured. ${ }^{22,23}$ In ref 48 we have shown that for mixtures of ethane and methane our model gives a reasonable prediction of the mixture isotherms. Here we concentrate on the mixtures of $\mathrm{C}_{4}$ through $\mathrm{C}_{7}$ isomers.

In Figures 23-26 the mixture isotherms of these isomers are presented. We focus on a mixture of a linear alkane and the 2-methyl isomer with a 50-50 mixture in the gas phase. For 


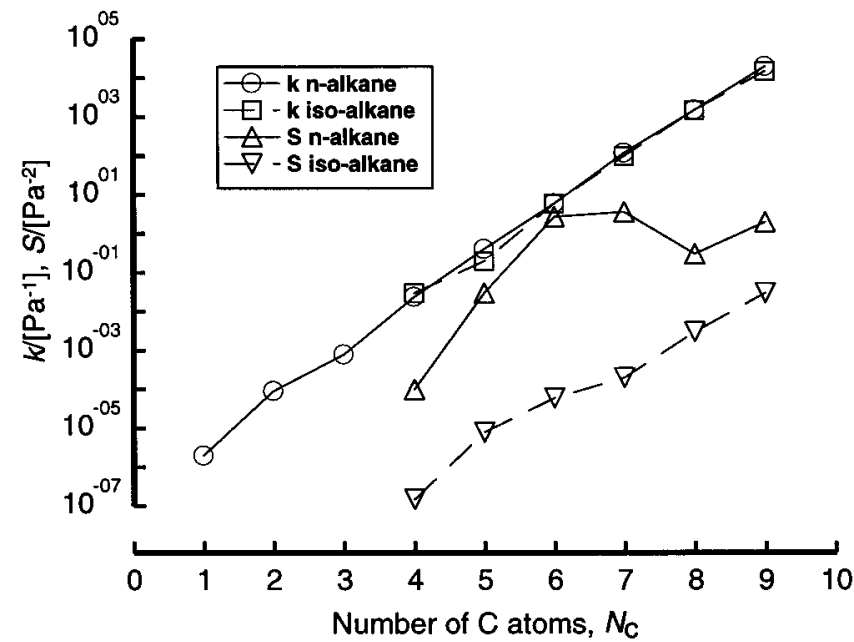

Figure 22. Parameters $k$ and $S$ of the dual-site Langmuir model for linear and branched alkanes as a function of the number of carbon atoms.

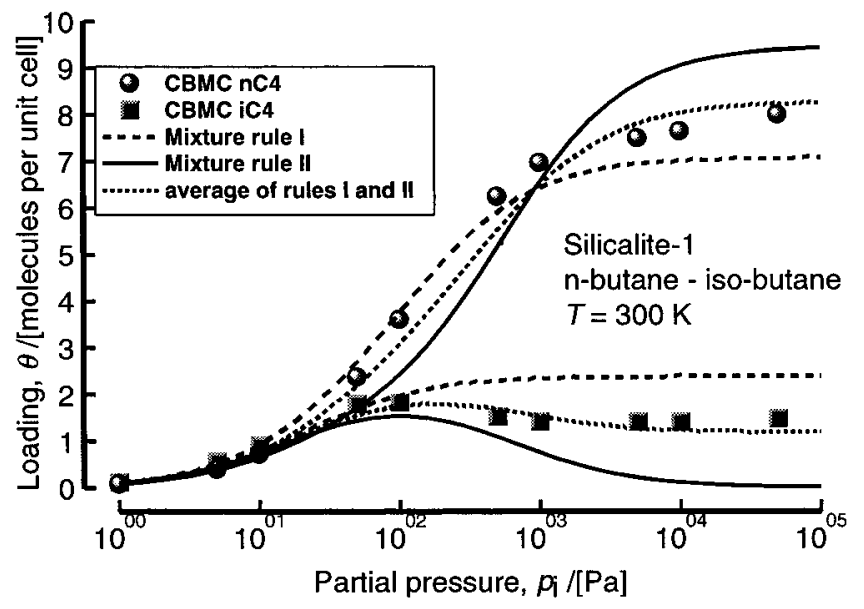

Figure 23. Adsorption isotherm of a 50-50 mixture of butane and isobutane in silicalite.

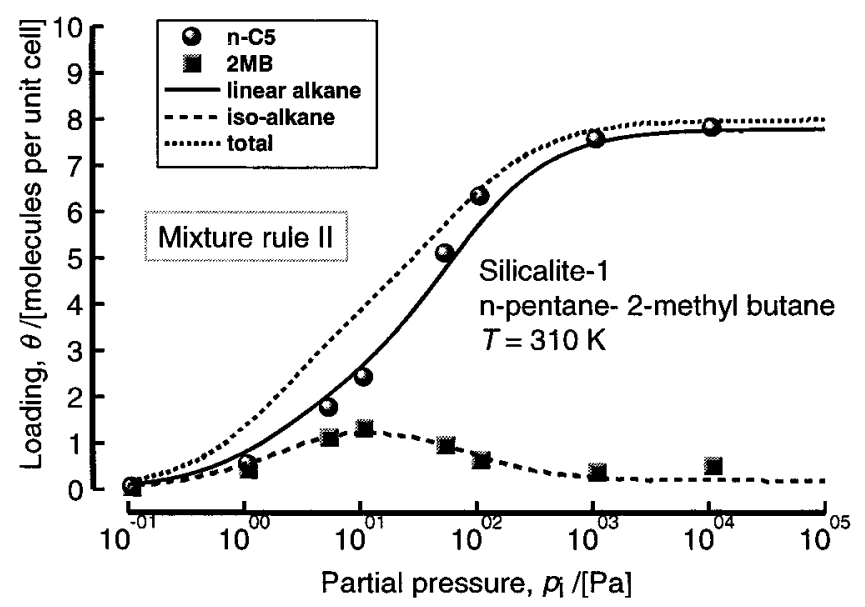

Figure 24. Adsorption isotherm of a 50-50 mixture of pentane and 2-methylbutane in silicalite.

all mixtures we see the following trends. At low pressure the linear and branched alkanes adsorb independently. The adsorption of the two components is proportional to the Henry coefficients of the pure components. At a total mixture loading of four molecules per unit cell the adsorption of the branched alkanes reaches a maximum and decreases with increasing pressure. The adsorption of the linear alkanes increases with increasing pressure until saturation is reached.

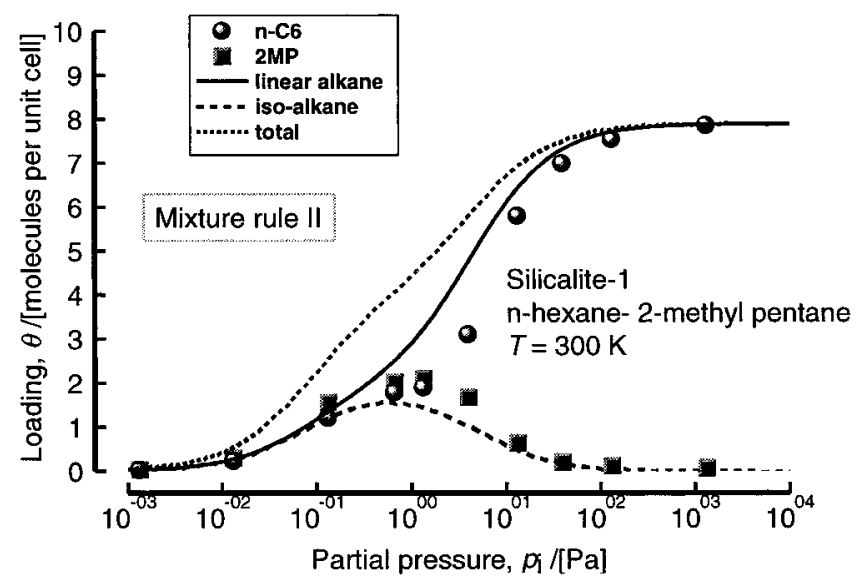

Figure 25. Adsorption isotherm of a 50-50 mixture of hexane and 2-methylpentane in silicalite.

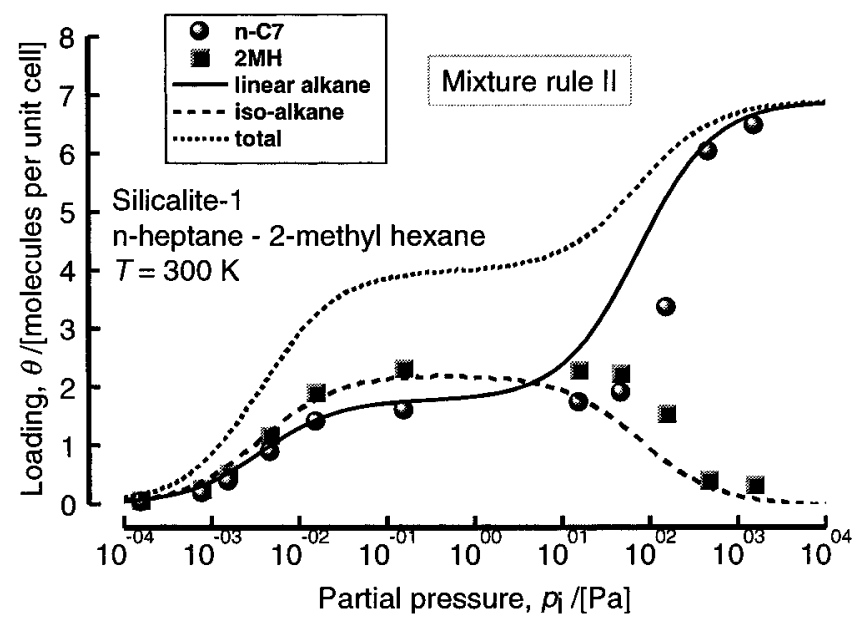

Figure 26. Adsorption isotherm of a 50-50 mixture of heptane and 2-methylhexane in silicalite.

There are also some qualitative differences between the various alkanes. The maximum in the isotherm of isobutane in the butane-isobutane mixture is very small, and at saturation the ratio of the loadings of butane and isobutane is approximately 5.6:1 (see Figure 23). For the pentane isomers the maximum is more pronounced and at saturation the concentration of the branched alkane is much lower, about one-sixth that of the linear alkane (see Figure 24). For the hexane isomers at maximum loading the branched alkane is completely squeezed out of the zeolite. For the heptane isomers a table-mountain maximum is observed (see Figure 26); here, too, the branched alkane is completely squeezed out at high pressures.

We see from Figures 24-26 that the simulated isotherms conform very well to the mixture rule II based on the dual-site Langmuir model. For alkanes with carbon atoms in the 5-7 range, we need to set up the mixture rule considering the total silicalite matrix (including sites A and B) as one entity. This is because the branched alkanes do not easily occupy site B (channel interiors) and for some pressure range the channel interiors are completely devoid of the branched isomers. The simulated isotherm for the 50-50 mixture of butane and isobutane behaves differently, however. Neither mixture rule, I or II, is completely successful. An average of the two mixture rules, on the other hand, is very successful. The reason that the $\mathrm{C}_{4}$ isomer mixture behaves differently from the $\mathrm{C}_{5}-\mathrm{C}_{7}$ isomers is because both sites $\mathrm{A}$ (intersections) and $\mathrm{B}$ (channel interiors) are accessible to both isomers over the 
whole pressure range. This is exactly what we observe in the simulations.

It is interesting to investigate the reasons why the branched alkanes are squeezed out by the linear alkanes at high pressures. For the $\mathrm{C}_{6}$ and $\mathrm{C}_{7}$ isomers the Henry coefficient of the branched alkanes is slightly larger. One would therefore expect that these branched alkanes would adsorb better. This is indeed observed at low pressures; at high pressures, however, other considerations have to be taken into account. We will explain this on the basis of the mixture behavior for $\mathrm{C}_{6}$ isomers. As can been seen from Figure 25 the total loading exhibits inflection behavior at $\theta_{1}+$ $\theta_{2}=4$. Until this loading there is no competition between $\mathrm{C}_{6}$ and 2-methylpentane (2MP) and both are almost equally easily adsorbed. Examination of the probability distributions of the linear and branched isomers at $100 \mathrm{~Pa}$ reveals that all the 2MP molecules are located at the intersections between the straight channels and the zigzag channels whereas $\mathrm{C}_{6}$ are located everywhere (see Figure 21). A further important aspect to note is the orientation of the 2MP molecules; these have their heads (i.e., the branched end) at the intersections and their tails sticking out into the zigzag or straight channels. The $\mathrm{C}_{6}$ molecules fit nicely into both straight and zigzag channels ${ }^{13}$ these molecules have a higher "packing efficiency" than 2MP. As the pressure is increased beyond $100 \mathrm{~Pa}$, it is more efficient to obtain higher loadings by "replacing" the $2 \mathrm{MP}$ with $\mathrm{C}_{6}$; this entropic effect is the reason behind the curious maximum in the $2 \mathrm{MP}$ isotherm. A similar explanation holds for the $\mathrm{C}_{5}$ and $\mathrm{C}_{7}$ isomers. To further test our hypothesis that for entropic reasons the branched alkanes are squeezed out of the zeolite, we have performed a simulation in which we have removed the attractive part of the LennardJones potential interacting between the hydrocarbon atoms and hydrocarbon-zeolite atoms. In such a system with only "hardsphere" interactions there is no energy scale involved and the only driving force is entropy. Also in this system we have observed that the branched alkane is squeezed out at high pressures, which proves that this squeezing out of the branched alkanes by the linear isomer is an entropic effect.

\section{Concluding Remarks}

The configurational-bias Monte Carlo technique (CBMC) has been used for simulating the adsorption isotherms for linear and branched 2-methylalkanes and their mixtures on silicalite. The important observations and conclusions arising from our studies are as follows:

(1) For branched alkanes inflection behavior was observed for all carbon numbers studied, which ranged from four to nine. This inflection was found to occur at a loading of four molecules per unit cell. Below this loading the branched alkanes are seen to be located predominantly at the intersections of the straight and zigzag channels. To obtain loadings higher than four, the branched alkane must seek residence in the channel interiors which is more demanding and therefore requires disproportionately higher pressures; this leads to the inflection behavior.

(2) Linear alkanes with six and more carbon atoms also were found to exhibit inflection behavior. Hexane and heptane show inflection due to commensurate "freezing"; the length of these molecules is commensurate with the length of the zigzag channels. This leads to a higher packing efficiency than for other linear alkanes.

(3) Available experimental data from the literature confirm the accuracy of the predictions of the CBMC simulations for both linear and branched alkanes. However, in the latter case the number of experimental data is much less as compared to that available for linear alkanes.
(4) The temperature dependency of the isotherms is also properly modeled by the CBMC simulations.

(5) For purposes of fitting the CBMC simulated isotherms,the dual-site Langmuir model has been found to provide an excellent description. In this model we distinguish between two sites with differing ease of adsorption: site A representing the intersections between the straight and zigzag channels, and site $B$ representing the channel interiors.

(6) CBMC simulations of isotherms of 50-50 binary mixtures of $\mathrm{C}_{5}, \mathrm{C}_{6}$, and $\mathrm{C}_{7}$ hydrocarbon isomers showed some remarkable and hitherto unreported features. The loading of the branched isomer in all three binary mixtures reaches a maximum when the total mixture loading corresponds to four molecules per unit cell. Higher loadings are obtained by "squeezing out" of the branched alkane from the silicalite and replacing these with the linear alkane. This "squeezing out" effect is found to be entropic in nature; the linear alkanes have a higher packing efficiency and higher loadings are more easily achieved by replacing the branched alkanes with the linear alkanes.

(7) The mixture isotherms can be predicted quite accurately by applying the appropriate mixture rule to the dual-site Langmuir model. This model allows the mixture isotherm to be predicted purely on the basis of the parameters describing the isotherms of the pure components, and the linear and branched alkane.

(8) The sorption selectivity exhibited by silicalite for the linear alkane in preference to the branched alkane in mixtures of $\mathrm{C}_{5}$, $\mathrm{C}_{6}$, and $\mathrm{C}_{7}$ hydrocarbon isomers provides a potential for the development of a novel separation technique based on entropydriven sorption selectivity. ${ }^{68}$

Acknowledgment. Financial support provided to T.J.H.V. from SON (Stichting Scheikundig Onderzoek Nederland) and to B.S. from the OSPT is acknowledged. This work was supported by the Netherlands Organisation for Scientific Research (NWO). A large part of the computer resources was generously provided by SARA (Stichting Academisch Rekencentrum Amsterdam). T.J.H.V. thanks Marcus G. Martin and J. Ilja Siepmann for instructive discussions on CBMC simulations of branched alkanes.

\section{Appendix A: Alkane Model}

In our study we focus on linear alkanes and branched alkanes with a single chain-end branch with the structure $\left(\mathrm{CH}_{3}\right)_{2}-\mathrm{CH}-$ $\left(\mathrm{CH}_{2}\right)_{N} \mathrm{CH}_{3}$. The pseudo-atoms in a given chain are assumed to be connected by rigid bonds $\left(d_{\mathrm{CC}}=1.53 \AA\right.$ ). Bond bending is modeled by a harmonic potential ${ }^{69}$

$$
u_{\text {bending }}\left(\theta_{i}\right)=(1 / 2) k_{\theta}\left(\theta_{i}-\theta_{\text {eq }}\right)^{2}
$$

with $\theta_{\mathrm{eq}}=113^{\circ}$ and the equilibrium angle for all hydrocarbons and with a force constant equal to $k_{\theta}=62500 \mathrm{~K} \mathrm{rad}^{-2}$. Changes in the torsional angles are controlled by. ${ }^{70}$

$$
u_{\text {torsion }}\left(\phi_{i}\right)=C_{0}+C_{1} \cos \left(\phi_{i}\right)+C_{2} \cos ^{2}\left(\phi_{i}\right)+C_{3} \cos ^{3}\left(\phi_{i}\right)
$$

with parameters shown in Table 2.

The pseudo-atoms in different molecules, or belonging to the same molecule, but separated by more than three bonds, interact which each other through a Lennard-Jones potential

$$
u_{i j}^{\mathrm{lj}}=4 \epsilon_{i j}\left[\left(\frac{\sigma_{i j}}{r_{i j}}\right)^{12}-\left(\frac{\sigma_{i j}}{r_{i j}}\right)^{6}\right]
$$



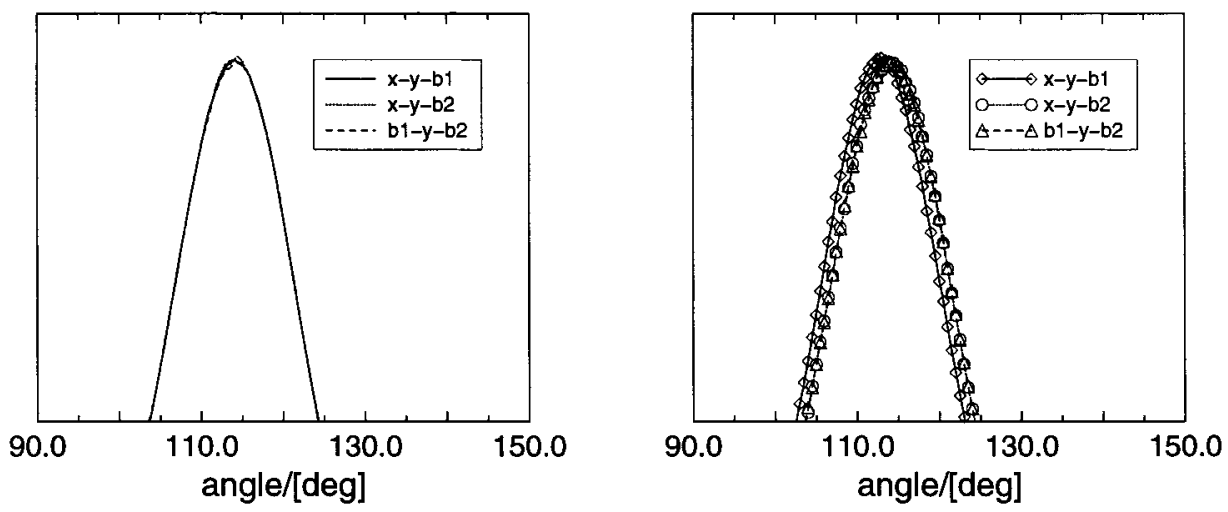

Figure 27. Part of the bond angle distributions at $T=1000 \mathrm{~K}$; (a) results of our algorithm and (b) the incorrect algorithm when the two beads are not inserted simultaneously $\left(d_{1}\right.$ always inserted before $\left.d_{2}\right)$. The differences between the distributions are approximately $2 \times 10^{-2}$ rad at $T=1000$ $\mathrm{K}$ and $3 \times 10^{-3} \mathrm{rad}$ at $T=300 \mathrm{~K}$.

where $r_{i j}$ is the distance between sites $i$ and $j$. We have used the Jorgensen mixing rules ${ }^{71}$

$$
\begin{gathered}
\epsilon_{i j}=\sqrt{\epsilon_{i} \epsilon_{j}} \\
\sigma_{i j}=\sqrt{\sigma_{i} \sigma_{j}}
\end{gathered}
$$

The Lennard-Jones potentials were truncated at $13.8 \AA$, and the usual tail corrections have been applied..$^{19,72}$ The LennardJones parameters used are shown in Table 3.

\section{Appendix B: Details on the Simulation Techniques}

In this Appendix we give a more detailed description of those aspects of the simulations that are different from what is published in the literature. In particular, in Section B1 we give the correct derivation of the reference state of a CBMC simulation in the grand-canonical ensemble. In Section B2 we discuss the way branched alkanes are generated in a CBMC move. We show that some of the schemes that have been published in the literature may lead to small errors.

B1. Reference State of the Chemical Potential. In a CBMC simulation of hydrocarbons it is convenient to split the potential into two parts: the internal interactions $\left(u^{\text {int }}\right)$ which include bond bending and torsion and external interactions $\left(u^{\mathrm{ext}}\right)$ which include the remainder of the interactions.

The internal interactions are used to generate a trial configuration and the external interactions are used to compute the Rosenbluth factor.

Assume that we perform an NVT simulation at infinite dilution and compute the normalized Rosenbluth factor of a chain with length $N_{c}$

$$
\mathrm{W}^{\mathrm{ext}}=\frac{1}{\mathrm{k}^{N_{c}-1}} \exp \left[-\beta u_{1}^{\mathrm{ext}}\right] \prod_{j=2}^{N_{c}} \sum_{i=1}^{k} \exp \left[-\beta u_{j}^{\mathrm{ext}}(i)\right]
$$

where $u_{j}(i)$ is the energy of atom $j$ at trial position $i, k$ is the number of trial positions, and $\beta=1 / k_{\mathrm{B}} T$. The $k$ trial positions are generated using the internal interactions

$$
p(b) \propto \exp \left[-\beta u^{\mathrm{int}}(b)\right]
$$

As is explained in refs 19 and 21, the use of the internal interactions to generate the trial positions causes a shift of the chemical potential in such a way that the average Rosenbluth factor is related to the difference of the chemical potential of the molecules $\mu$ and the chemical potential of an ideal chain $\mu_{\text {IC }}$

$$
\left\langle\mathrm{W} \quad{ }^{\mathrm{ext}}\right\rangle=\exp \left[-\beta\left(\mu-\mu_{\mathrm{IC}}\right)\right]
$$

An ideal chain is defined as a chain having internal interactions only. Important to note is that an ideal chain is different from a "real" chain in the ideal gas phase. For butane all interactions within a molecule are included in the internal interactions, but for pentane and the longer alkanes the Lennard-Jones interactions between, for example, beads 1 and 5 are not in the internal but in the external interactions. From a separate simulation of an isolated molecule, one can compute the Rosenbluth factor of an ideal gas molecule

$$
\begin{aligned}
\langle\mathrm{W} & \underset{\mathrm{IG}}{\mathrm{ext}}\rangle=\exp \left[-\beta\left(\mu_{\mathrm{IG}}-\mu_{\mathrm{IC}}\right)\right]= \\
& \frac{1}{k^{N_{c}-1}} \exp \left[-\beta u_{1}^{\mathrm{ext}}\right] \prod_{j=2}^{N_{c}} \sum_{i=1}^{k} \exp \left[-\beta u_{j}^{\mathrm{ext}}(i)\right]
\end{aligned}
$$

where $\mu_{\mathrm{IG}}$ is the chemical potential of an ideal gas of chain molecules. For the normalized Rosenbluth factor we can write

$$
\begin{aligned}
\langle\mathrm{W} \underset{\mathrm{IC}}{\mathrm{ext}}\rangle & =\exp \left[-\beta\left(\mu-\mu_{\mathrm{IG}}\right)+\beta\left(\mu_{\mathrm{IC}}-\mu_{\mathrm{IG}}\right)\right] \\
& =\exp \left[-\beta\left(\mu^{\mathrm{ex}}-\mu_{\mathrm{IC}}^{\mathrm{ex}}\right)\right]
\end{aligned}
$$

This equation shows that if we use the internal energy to generate the trial configuration, the average Rosenbluth factor is related to a shifted excess chemical potential.

Similarly, for a grand-canonical Monte Carlo simulation. In such a simulation we ensure that the chemical potential of the molecules in the reservoir $\left(\mu_{\mathrm{B}}\right)$ and the zeolite are equal, ${ }^{19}$ i.e., the average Rosenbluth factors in these two systems are equal. To compute the chemical potential we have to add this shift to the chemical potential of the reservoir $\left(\mu_{\mathrm{B}}\right)$ which is used in the acceptance rules:

$$
\beta \mu=\beta \mu_{\mathrm{B}}+\beta \mu_{\mathrm{IC}}^{\mathrm{ex}}
$$

This shift has to be recalculated for each temperature. In ref 36 this shift was given incorrectly a minus sign.

B2. Generation of Branched Alkanes. The following strategies have been used to grow branched molecules. Siepmann et al., ${ }^{27}$ and Zhuravlev and Siepmann ${ }^{73}$ have used a fixed growth path along the molecule, Cui et al. ${ }^{74}$ first grow the backbone of a molecule and then insert the side-chains, and Dijkstra $^{75}$ grows all groups at a branch simultaneously. The first two approaches implicitly assume that branches connected to the same central atom can be added independently. Below we demonstrate that, because of the presence of bond-bending 
TABLE 4: Experimental Heat of Adsorption of Various Alkanes $\left(q_{\mathrm{st}}\right)$ in Silicalite/ZSM5

\begin{tabular}{|c|c|c|c|c|c|c|c|}
\hline$T / K$ & $\mathrm{Si} / \mathrm{Al}$ ratio & $-q_{\mathrm{st}} /(k J / m o l)$ & ref & $T / K$ & $\mathrm{Si} / \mathrm{Al}$ ratio & $-q_{\mathrm{st}} /(\mathrm{kJ} / \mathrm{mol})$ & ref \\
\hline \multicolumn{4}{|c|}{ Methane } & \multicolumn{4}{|c|}{ Butane } \\
\hline 300 & $\infty$ & 18.1 & 87,88 & 293 & 34 & 49.5 & 76 \\
\hline 300 & $\infty($ Linde S-115) & 20.4 & 22 & 300 & $\infty$ & 48.7 & 85 \\
\hline 300 & $\infty$ & 20.5 & 80 & 300 & $\infty$ & 51 & 18 \\
\hline 300 & $\infty$ & 20.0 & 50 & 300 & $\infty$ & 50.4 & 80 \\
\hline 300 & $\infty$ & 20.92 & 89,78 & 303 & 132 & $50_{1}$ & 6 \\
\hline 300 & $\infty$ & 20 & 16 & 301 & $\infty$ & $54.8_{1}$ & $84,94,97$ \\
\hline 300 & $>3000$ & 18.6 & 14 & 325 & $\infty($ Linde S-115) & 48.3 & 22 \\
\hline 300 & 52 & 28 & 90 & 400 & 10 (Na,ZSM5) & 55 & 98 \\
\hline 300 & $\infty$ & 18.7 & 81 & 400 & 10 (H,ZSM5) & 53 & 98 \\
\hline 300 & $\infty$ & 20.9 & 91 & 400 & 24 (Na,ZSM5) & 52 & 98 \\
\hline \multirow[t]{3}{*}{423} & $\infty$ & 22.5 & 92 & 400 & 24 (H,ZSM5) & 50 & 98 \\
\hline & & & & 400 & 44 (Na,ZSM5) & 50 & 98 \\
\hline & & & & 400 & $\infty$ & 48 & 98 \\
\hline 293 & 34 & 40.0 & 76 & 423 & $\infty$ & 49.5 & 92 \\
\hline 293 & 130 & 45 & 76 & 300 & $\infty$ & 51 & 16 \\
\hline 298 & $\infty$ & 30.5 & 83 & 300 & $>3000$ & 53.0 & 14 \\
\hline 300 & $\infty$ & 31.1 & 91 & & & & \\
\hline 300 & 30 (Na,ZSM5) & 38.0 & 93 & \multicolumn{4}{|c|}{ Pentane } \\
\hline 300 & $\infty($ Linde $S-115)$ & 32.8 & 22 & 293 & 34 & 54 & 76 \\
\hline 300 & $\infty$ & 29.9 & 80 & 303 & $\infty$ & $64.5_{4}$ & 4,5 \\
\hline 300 & $\infty$ & 31 & 18 & 300 & $\infty$ & 41.8 & 9 \\
\hline 300 & $\infty$ & 33 & 16 & & & & \\
\hline 300 & $>>3000$ & 30.7 & 14 & \multicolumn{4}{|c|}{ Hexane } \\
\hline 301 & $\infty$ & 34 & 94 & 300 & $\infty$ & $71_{4}$ & 99 \\
\hline 318 & 1230 & 30 & 95 & 333 & 132 & $60_{1}$ & 6 \\
\hline \multirow[t]{3}{*}{333} & 132 & 30 & 6 & 300 & $\infty$ & $71.5_{1}$ & 100,2 \\
\hline & & & & 300 & $\infty$ & 71 & 18 \\
\hline & & & & 318 & $135(\mathrm{Na}, \mathrm{H}-\mathrm{ZSM} 5)$ & 71 & 95 \\
\hline 300 & $\infty$ & 42.2 & 96 & 300 & $\infty$ & 70.5 & 9 \\
\hline 293 & 34 & 44.5 & 76 & & & & \\
\hline 293 & 130 & 46.5 & 76 & \multicolumn{4}{|c|}{ Heptane } \\
\hline 298 & $\infty$ & 38 & 83 & 303 & $\infty$ & $84.5_{3}$ & 4,5 \\
\hline 300 & $\infty$ & 40.7 & 80 & 300 & $\infty$ & 83.4 & 9 \\
\hline 300 & $\infty$ & 40 & 16 & & & & \\
\hline 300 & $>3000$ & 40.9 & 14 & \multicolumn{4}{|c|}{ Octane } \\
\hline 325 & $\infty($ Linde S-115) & 39.9 & 22 & 300 & $\infty$ & 92.1 & 9 \\
\hline 318 & 1230 & 40 & 95 & & & & \\
\hline 318 & $135(\mathrm{Na}, \mathrm{H}-\mathrm{ZSM} 5)$ & 36.7 & 95 & \multicolumn{4}{|c|}{ Nonane } \\
\hline 333 & 132 & 39 & 6 & 300 & $\infty$ & 107.7 & 9 \\
\hline \multirow[t]{5}{*}{423} & $\infty$ & 36.5 & 92 & & & & \\
\hline & & & & \multicolumn{4}{|c|}{ Decane } \\
\hline & & & & 300 & $\infty$ & 112 & 18 \\
\hline & & & & 303 & $\infty$ & $110.5_{4}$ & 2 \\
\hline & & & & 300 & $\infty$ & 120.5 & 9 \\
\hline
\end{tabular}

potentials, this assumption is not valid for the hydrocarbons studied by these authors.

Let us consider the growing of isobutane which is the simplest case of a branched alkane. Assume that we have already inserted the first two segments $\mathbf{x}, \mathbf{y}$ using the conventional growing schemes. We now have to add the following two segments $\left(\mathbf{b}_{1}\right.$, $\mathbf{b}_{2}$ ) that are connected to segment $\mathbf{y}$. We have to generate the position of a trial set $\mathbf{B}=\left(\mathbf{b}_{1}, \mathbf{b}_{2}\right)$ where $\mathbf{b}_{1}$ and $\mathbf{b}_{2}$ are the trial positions of the two atoms of the branch that will be added. In the CBMC scheme the a probability of this set is proportional to its Boltzmann weight: ${ }^{19}$

$$
p(\mathbf{B}) \mathrm{d} \mathbf{B} \propto \exp \left[-\beta u_{\text {bend }}(\mathbf{B})\right] \mathrm{d} \mathbf{B}
$$

in which $u_{\text {bend }}$ is the total bond-bending energy:

$$
u_{\text {bend }}(\mathbf{B})=u_{\text {bend }}\left(\mathbf{x}, \mathbf{y}, \mathbf{b}_{1}\right)+u_{\text {bend }}\left(\mathbf{x}, \mathbf{y}, \mathbf{b}_{2}\right)+u_{\text {bend }}\left(\mathbf{b}_{1}, \mathbf{y}, \mathbf{b}_{2}\right)
$$

From these equations it follows that the probability of generating $\mathbf{B}$ is equal to the probability of generating position $\mathbf{b}_{1}$ multiplied by the probability of generating $\mathbf{b}_{2}$ under the condition of having generated $\mathbf{b}_{1}$ already:

$$
p(\mathbf{B})=p\left(\mathbf{b}_{1}\right) p\left(\mathbf{b}_{2} \mid \mathbf{b}_{1}\right)
$$

In the schemes used by Siepmann and co-workers ${ }^{27,73}$ and Cui et al. ${ }^{74}$ it is assumed that

$$
p\left(\mathbf{b}_{1}\right) \propto \exp \left[-\beta u_{\text {bend }}\left(\mathbf{x}, \mathbf{y}, \mathbf{b}_{1}\right)\right]
$$

and

$$
p\left(\mathbf{b}_{2} \mid \mathbf{b}_{1}\right) \propto \exp \left[-\beta\left(u_{\text {bend }}\left(\mathbf{x}, \mathbf{y}, \mathbf{b}_{2}\right)+u_{\text {bend }}\left(\mathbf{b}_{1}, \mathbf{y}, \mathbf{b}_{2}\right)\right)\right]
$$

It is important to note that this is assumption is only valid if

$$
\begin{aligned}
\left\{\int \mathrm{d} \mathbf{b}_{1} \mathrm{e}^{-\beta u_{\text {bend }}\left(\mathbf{x}, \mathbf{y}, \mathbf{b}_{1}\right)}\right\}\left\{\int \mathrm{d} \mathbf{b}_{2} \mathrm{e}^{-\beta\left[u_{\text {bend }}\left(\mathbf{x}, \mathbf{y}, \mathbf{b}_{2}\right)+u_{\text {bend }}\left(\mathbf{b}_{1}, \mathbf{y}, \mathbf{b}_{2}\right)\right]}\right\}= \\
\left\{\int \mathrm{d} \mathbf{b}_{1} \mathrm{e}^{-\beta u_{\text {bend }}\left(\mathbf{x}, \mathbf{y}, \mathbf{b}_{1}\right)} \int \mathrm{d} \mathbf{b}_{2} \mathrm{e}^{-\beta\left[u_{\text {bend }}\left(\mathbf{x}, \mathbf{y}, \mathbf{b}_{2}\right)+u_{\text {bend }}\left(\mathbf{b}_{1}, \mathbf{y}, \mathbf{b}_{2}\right)\right]}\right\}
\end{aligned}
$$

Because of the dependence of the second term on the left-hand side on $\mathbf{b}_{2}$ this equation does not hold in general.

This suggests that if this scheme is used in a simulation the three angle distributions may not be identical. In Figure 27 it is shown that this is indeed the case. Important to note is that this difference is observed only if the temperature is very large. We therefore expect that at the conditions simulated in refs. ${ }^{27,73,74}$ the differences between the various angle distributions will be much smaller and therefore will not influence the end results. 
The scheme used by Dijkstra ${ }^{75}$ in which both atoms are generated simultaneously does not suffer from this problem. In this work we have used a Monte Carlo scheme to generate the trial positions simultaneously. This means that we perform a small MC simulation within a MC simulation itself. The number of Monte Carlo steps between samples is chosen sufficiently large that correlations between two sets of trial positions are negligible. The maximum displacements are chosen such that the autocorrelation functions of the bond angles and torsion angles decay to zero within a minimum number of trial moves. In addition one should be careful that all torsions will be sampled.

\section{Appendix C: Discussion of the Experimental Data}

Heats of Adsorption. In our model we have used the heats of adsorption and the Henry coefficients to fit our model. Unfortunately, these is significant scatter in the experimental data which makes it difficult to refer to the literature for the experimental data. In our comparison with the simulation results, we have made a selection of the experimental data. Appendix $\mathrm{C}$ provides a short justification of this selection. The available experimental data for the heats of adsorption are summarized in Table 4.The experimental data for pure silicalite are in the range -18 to $-22 \mathrm{~kJ} / \mathrm{mol}$. In our simulations we have used $-20 \mathrm{~kJ} / \mathrm{mol}$. For ethane the experimental data on pure silicalite converge to a value of $-31 \mathrm{~kJ} / \mathrm{mol}$ and for propane to a value of $-40 \mathrm{~kJ} / \mathrm{mol}$. For the longer alkanes we have used $-50 \mathrm{~kJ} /$ mol for butane. For pentane the data scatter significantly. The data reported by Sun et al. ${ }^{9}$ suggests that the heat of adsorption of pentane is lower than the heat of adsorption of hexane also the data in refs $\mathrm{s}^{4,5,76}$ do not give a consistent result. We have used $-60 \mathrm{~kJ} / \mathrm{mol}$ which is consistent with the data for butane and hexane. For hexane the experimental data agree much better. These data converge well to a value of $-71 \mathrm{~kJ} / \mathrm{mol}$. For the longer alkanes only a few data have been published which makes it difficult to compare the consistency. We have used for heptane $-83 \mathrm{~kJ} / \mathrm{mol}$, for octane $-92 \mathrm{~kJ} / \mathrm{mol}$, for nonane $-108 \mathrm{~kJ} / \mathrm{mol}$, and for decane $-120 \mathrm{~kJ} / \mathrm{mol}$.

Henry Coefficients. Adsorption isotherms of methane in silicalite have been determined by several groups. ${ }^{22,50,77-81}$ At low pressures the data from Hufton and Danner, ${ }^{80}$ Yamazaki et al., ${ }^{78}$ Ott et al., ${ }^{79}$ Rees et al., ${ }^{50}$ and Golden and Sircar ${ }^{81}$ are in very good agreement. From these adsorption isotherms we have determined the Henry coefficients and we have used $H=7.5$ $\times 10^{-6} \mathrm{mmol} \mathrm{g}^{-1} \mathrm{~Pa}^{-1}$ as the experimental value for the Henry coefficient. For ethane the data of Hufton and Danner, ${ }^{80,82}$ Richard and Rees, ${ }^{6}$ and Hampson and Rees ${ }^{83}$ are in good agreement with each other. We have combined the low-pressure data of Hufton and Danner, ${ }^{80,82}$ Richard and Rees, ${ }^{6}$ and Hampson and Rees. ${ }^{83}$ Fitting all these data with equal weight yielded a Henry coefficient $H=1.4 \times 10^{-4} \mathrm{mmol}^{-1} \mathrm{~g}^{-1} \mathrm{~Pa}^{-1}$. This is consistent with the values reported in refs 80 and 83 . The adsorption isotherms of propane of Abdul-Rehman et al. ${ }^{22}$ and Hampson and Rees ${ }^{83}$ are in good agreement with each other. The data of Richard and Rees ${ }^{6}$ deviate slightly. Note that the isotherm of Richard and Rees was measured at a temperature of $291.5 \mathrm{~K}$, while the other data are taken at $300 \mathrm{~K}$. This temperature difference can explain the difference between the data sets. In our calculations, we have used $H=1.25 \times 10^{-3}$ mmol g ${ }^{-1} \mathrm{~Pa}^{-1}$. For butane, isotherms have been measured by Thamm, ${ }^{84}$ Stach et al., ${ }^{2}$ Richard and Rees, ${ }^{6}$ Abdul-Rehman, ${ }^{22}$ and Shen and Rees. ${ }^{85}$ These isotherms gave a Henry coefficient of approximately $1.5 \times 10^{-2} \mathrm{mmol}^{-1} \mathrm{~g}^{-1} \mathrm{~Pa}^{-1}$. Adsorption isotherms of pentane in silicalite have been measured by
Rakhmatkariev et al. ${ }^{4}$ and Dubinin et al. ${ }^{5}$ Dubinin et al. ${ }^{5}$ report data at low pressures yielding a Henry coefficient of 0.187 $\mathrm{mmol}^{-1} \mathrm{~g}^{-1} \mathrm{~Pa}^{-1}$. For hexane, adsorption isotherms have been measured by Stach et al. ${ }^{2}$ and Richard and Rees. ${ }^{6}$ We have used the average of the two Henry coefficients, namely $3.05 \mathrm{mmol}^{-1}$ $\mathrm{g}^{-1} \mathrm{~Pa}^{-1}$. For the longer alkanes we could not find sufficiently reliable isotherms at low pressures to compute a Henry coefficient at room temperature.

\section{References and Notes}

(1) Haag, W. O. In Zeolites and Related Microporous Materials: State of the Art 1994, Studies in Surface Science and Catalysis; Weitkamp, J., Karge, H. G., Pfeifer, H., Hölderich, W., Eds.; Elsevier: Amsterdam, 1994; Vol. 84, pp 1375-1394.

(2) Stach, H.; Lohse, U.; Thamm, H.; Schirmer, W. Zeolites 1986, 6, 74-90.

(3) Lohse, U.; Fahlke, B. Chem. Techn. 1983, 35, 350-353.

(4) Rakhmatkariev, G. U.; Zhalalov, Kh. R.; Akhmedov, K. S. Uzb. Khim. Zh. 1988, 3, 68-70.

(5) Dubinin, M. M.; Rakhmatkariev, G. U.; Isirikyan, A. A. Izv. Akad. Nauk SSSR, Ser. Khim. 1989, 10, 2333-2335.

(6) Richard, R. E.; Rees, L. V. C. Langmuir 1987, 3, 335-340.

(7) Well, W. J. M. van; Wolthuizen, J. P.; Smit, B.; Hooff, J. H. C. van; Santen, R. A. van. Angew. Chem., Int. Ed. 1995, 34, 2543-2544.

(8) Olsen, D. H.; Reischmann, P. T. Zeolites 1996, 17, 434-436. 17280

(9) Sun, M. S.; Talu, O.; Shah, D. B. J. Phys. Chem. 1996, 100, 17276-

(10) Yang, Y.; Rees, L. V. C. Microporous Matter. 1997, 12, 117122.

(11) Yang, Y.; Rees, L. V. C. Microporous Matter. 1997, 12, 223-

(12) Millot, B.; Methivier, A.; Jobic, H. J. Phys. Chem. B 1998, 102, $3210-3215$

(13) Smit, B.; Maesen, T. L. M. Nature 1995, 374, 42-44.

(14) Zhu, W.; Graaf, J. M. van de; Broeke, L. J. P. van den; Kapteijn, F.; Moulijn, J. A. Ind. Eng. Chem. Res. 1998, 37, 1934-1942.

(15) Vlugt, T. J. H.; Zhu, W.; Moulijn, J. A.; Smit, B.; Krishna, R. J. Am. Chem. Soc. 1998, 120, 5599-5600.

(16) Sun, M. S.; Shah, D. B.; Xu, H. H.; Talu, O. J. Phys. Chem. 1998, $102,1466-1473$

(17) Cavalcante, C. L., Jr.; Ruthven, D. M. Ind. Eng. Chem. Rev. 1995, $34,177-184$.

(18) Stach, H.; Thamm, H.; Jänchen, J.; Fiedler, K.; Schirmer, W. In New Developments in Zeolite Science and Technology, Proceedings of the 6th International Zeolite Conference; Olsen, D., Bisio, A., Eds.; Butterworth: Guildford, U.K., 1984; pp 225-231.

(19) Frenkel, D.; Smit, B. Understanding Molecular Simulations: from Algorithms to Applications; Academic Press: San Diego, 1996.

(20) Smit, B.; Siepmann, J. I. Science 1994, 264, 1118-1120.

(21) Smit, B.; Siepmann, J. I. J. Phys. Chem. 1994, 98, 8442-8452.

(22) Abdul-Rehman, H. B.; Hasanain, M. A.; Loughlin, K. F. Ind. Eng. Chem. Res. 1990, 29, 1525-1535.

(23) Dunne, J. A.; Rao, M.; Sircar, S.; Gorte, R. J.; Myers, A. L. Langmuir 1997, 13, 4333-4341.

(24) Ryckaert, J. P.; Bellemans, A. Faraday Discuss. Chem. Soc. 1978, 66, 95-106.

(25) Smit, B.; Karaborni, S.; Siepmann, J. I. J. Chem. Phys. 1995, 102, 2126-2140; erratum: J. Chem. Phys. 1998, 109, 352.

(26) Siepmann, J. I.; Karaborni, S.; Smit, B. J. Am. Chem. Soc. 1993, $115,6454-6455$.

(27) Siepmann, J. I.; Martin, M. G.; Mundy, C. J.; Klein, M. L. Mol. Phys. 1997, 90, 687-693.

(28) Martin, M. G.; Siepmann, J. I. J. Phys. Chem. B 1998, 102, 25692577.

(29) Bezus, A. G.; Kiselev, A. V.; Lopatkin, A. A.; Du, P. Q. J. Chem. Soc., Faraday Trans. 2 1978, 74, 367-379.

(30) June, R. L.; Bell, A. T.; Theodorou, D. N. J. Phys. Chem. 1992, 96, 1051-1060

(31) Smit, B. J. Phys. Chem. 1995, 99, 5597-5603.

(32) Siepmann, J. I.; Frenkel, D. Mol. Phys. 1992, 75, 59-70.

(33) Frenkel, D.; Mooij, G. C. A. M.; Smit, B. J. Phys.: Condens. Matter 1992, 4, 3053-3076.

(34) Pablo, J. J.de; Laso, M.; Suter, U. W. J. Chem. Phys. 1992, 96, $6157-6162$

(35) Siepmann, J. I. In Computer simulation of biomolecular systems: theoretical and experimental applications; Gunsteren, W. F. van; Weiner, P. K.; Wilkinson, A. J., Eds.; Escom Science Publisher: Leiden, 1993; pp $249-264$.

(36) Smit, B. Mol. Phys. 1995, 85, 153-172. 
(37) Bates, S. P.; Well, W. J. M. van; Santen, R. A. van; Smit, B. J. Am. Chem. Soc. 1996, 118, 6753-6759.

(38) Bates, S. P.; Well, W. J. M. van; Santen, R. A. van; Smit, B. J. Phys. Chem. 1996, 100, 17573-17581.

(39) Bandyopadhyay, S.; Yashonath, S. J. Phys. Chem. B 1997, 101, $5675-5683$

(40) Well, W. J. M. van; Cottin, X.; Haan, J. W. de; Santen, R. A. van; Smit, B. Angew. Chem., Int. Ed. 1998, 37, 1081-1083.

(41) Well, W. J. M. van; Cottin, X.; Smit, B.; Hooff, J. H. C. van; Santen, R. A. van. J. Phys. Chem. B 1998, 102, 3952-3958.

(42) Martin, M. G.; Siepmann, J. I. J. Am. Chem. Soc. 1997, 119, 89218924.

(43) Panagiotopoulos, A. Z. Int. J. Thermophys. 1989, 10, 447.

(44) Esselink, K.; Loyens, L. D. J. C.; Smit, B. Phys. Rev. E 1995, 51, $1560-1568$.

(45) Vlugt, T. J. H.; Martin, M. G.; Smit, B.; Siepmann, J. I.; Krishna, R. Mol. Phys. 1998, 94, 727-733.

(46) http://molsim.chem.uva.nl/bigmac/.

(47) Maginn, E. J.; Bell, A. T.; Theodorou, D. N. J. Phys. Chem. 1995, 99, 2057-2079

(48) Du, Z.; Vlugt, T. J. H.; Smit, B.; Manos, G. AIChE J. 1998, 44, $1756-1764$

(49) The data in ref 22 have been corrected for the binder by assuming that this binder does not contribute to the adsorption.

(50) Rees, L. V. C.; Brückner, P.; Hampson, J. Gas Sep. Purif. 1991, 5 , $67-75$.

(51) Gregg, S. J.; Sing, K. S. W. Adsorption, Surface Area and Porosity; Academic Press: London, 1982.

(52) Evans, R. In Liquides aux Interfaces/Liquids at interfaces, Les Houches, Session XLVIII, 1988; by Charvolin, J., Joanny, J. F., Zinn-Justin, J., Eds.; North Holland: Amsterdam, 1990; pp 1-98. 850.

(53) Radhkrishnan, R.; Gubbins, K. E. Phys. Rev. Lett. 1997, 79, $2847-$

(54) Maris, T.; Vlugt, T. J. H.; Smit, B. J. Phys. Chem. B 1998, 102, $7183-7189$

(55) Recently, there is a discussion in the literature whether pseudoone-dimensional phase transitions can occur in molecular sieves (refs 53 $54)$

(56) Recent simulations by Du et al ${ }^{48}$ indicate that for ethane at high pressure also an inflection may be observed. However, experimental evidence for this inflection is still lacking.

(57) Lohse, U.; Thamm, H.; Noack, M.; Fahlke, B. J. Inclusion Phenom. 1987, 5, 307-313.

(58) Eder, F. Thermodynamics and siting of alkane sorption in molecular sieves. Ph.D. Thesis, Universiteit Twente, 1996.

(59) Eder, F.; Lercher, J. A. Zeolites 1997, 8, 75-81.

(60) Eder, F.; Lercher, J. A. J. Phys. Chem. B 1997, 101, 1273-1278.

(61) Eder, F.; Stockenhuber, M.; Lercher, J. A. J. Phys. Chem. B 1997, $101,5414-5419$

(62) Eder, F.; Lercher, J. A. Unpublished data (used with permission of the authors)

(63) June, R. L.; Bell, A. T.; Theodorou, D. N. J. Phys. Chem. 1990, $94,1508-1516$

(64) Smit, B.; Loyens, L. D. J. C.; Verbist, G. L. M. M. Faraday Discuss. 1997, 106, 93-104.

(65) Micke, A.; Bülow, M.; Kocirik, M.; Struve, P. J. Phys. Chem. 1994 $98,12337-12344$.

(66) Ruthven, D. M. In Principles of adsorption and adsorption processes; John Wiley: New York, 1984; p 91.

(67) Sung, W.; Park, P. J. Phys. Rev. Lett. 1996, 77, 783-786.

(68) Krishna, R.; Smit, B.; Vlught, T. J. H. J. Phys. Chem. A 1998, $102,7727-7730$.
(69) Ploeg, P. Van der; Berendsen, H. J. C. J. Chem. Phys. 1982, 76, $3271-3276$

(70) Wang, Y.; Hill, K.; Harris, J. G. J. Phys. Chem. 1994, 100, 32763285 .

(71) Jorgensen, W. L.; Madura, J. D.; Swenson, C. J. J. Am. Chem. Soc. 1984, 106, 6638-6646.

(72) Allen, M. P.; Tildesley, D. J. Computer Simulation of Liquids; Clarendon Press: Oxford, 1987.

(73) Zhuravlev, N. D.; Siepmann, J. I. Fluid Phase Equilibria 1997, $134,55-61$

(74) Cui, S. T.; Cummings, P. T.; Cochran, H. D. Fluid Phase Equilib. 1997, 141, 45-61.

(75) Dijkstra, M. J. Chem. Phys. 1997, 107, 3277-3288.

(76) Doelle, H.-J.; Heering, J.; Riekert, L.; Marosi, L. J. Catal. 1981, $71,27-40$

(77) Ding, T.; Ozawa, S.; Ogino, Y. Zhejiang Daxue Xuebao 1988, 22,

(78) Yamazaki, T.; Watanuki, I.; Ozawa, S.; Ogino, Y. Langmuir 1988, $4,433-438$

(79) Otto, K.; Montreuil, C. N.; Todor, O.; McCabe, R. W.; Gandhi, H. S. Ind. Eng. Chem. Res. 1991, 30, 2333-2340.

(80) Hufton, J. R.; Danner, R. P. AIChE J. 1993, 39, 954-961.

(81) Golden, T. C.; Sircar, S. J. Colloid Interface Sci. 1989, 162, 182188

(82) Hufton, J. R.; Danner, R. P. Chem. Eng. Sci. 1991, 46, 20792091.

(83) Hampson, J. A.; Rees, L. V. C. J. Chem. Soc., Faraday Trans. 1993, 89, 3169-3176.

(84) Thamm, H.; Stach, H.; Schirmer, W.; Fahlke, B. Z. Phys. Chem. (Leipzig) 1982, 263, 461-469.

(85) Shen, D.; Rees, L. V. Zeolites 1991, 119, 684-689.

(86) Verlet, L.; Weis, J. J. Mol. Phys. 1972, 24, 1013-1024.

87) Hyun, S. H.; Danner, R. P. AIChE Symp. Ser. 1982, 78(219), 19-

(88) Hufton, J. R. J. Phys. Chem. 1991, 95, 8836-8839

(89) Yamazaki, T.; Watanuki, I.; Ozawa, S.; Ogino, Y. Nippon Kagaku Kaishi 1987, 8, 1535-1540.

(90) Papp, H.; Hinsen, W.; Do, N. T.; Baerns, M. Therm. Acta 1984 $82,137-148$

(91) Dunne, J. A.; Mariwala, R.; Rao, M.; Sircar, S.; Gorte, R. J.; Myers, A. L. Langmuir 1996, 12, 5888-5895.

(92) Chiang, A. S.; Dixon, A. G.; Ma, Y. H. Chem. Eng. Sci. 1984, 39, $1461-1468$.

(93) Dunne, J. A.; Rao, M.; Sircar, S.; Gorte, R. J.; Myers, A. L. Langmuir 1996, 12, 5896-5904.

(94) Thamm, H. Zeolites 1987, 7, 341-346.

(95) Bülow, M.; Schodder, H.; Rees, L. V. C.; Richards, R. E. In New Developments in Zeolite Science and Technology, Proceedings of the 7th International Zeolite Conference; Murakami, Y., Iijima, A., Ward, J. W., Eds.; Elsevier: Amsterdam, 1986; pp 579-586.

(96) Kiselev, A. V.; Lopatkin, A. A.; Shulga, A. A. Zeolites 1985, 5 , 261-267.

(97) Thamm, H.; Stach, H.; Fiebig, W. Zeolites 1983, 3, 95-97.

(98) Lechert, H.; Schweiter, W. In New Developments in Zeolite Science and Technology, Proceedings of the 6th International Zeolite Conference; Olsen, D., Bisio, A., Eds.; Butterworth: Guildford, U.K., 1984; pp 210216.

(99) Flanigen, E. M.; Bennett, J. M.; Grose, R. W.; Cohen, J. P.; Patton, R. L.; Kirchener, R. M.; Smith, J. V. Nature 1978, 271, 512-516.

(100) Thamm, H.; Regent, N. I. Z. Chem. 1982, 22, 232-233. 